\title{
Entrainment and chaos in a pulse-driven Hodgkin-Huxley oscillator
}

\author{
Kevin K. Lin \\ klinecims.nyu.edu \\ January 18, 2006
}

\begin{abstract}
The Hodgkin-Huxley model describes action potential generation in certain types of neurons and is a standard model for conductance-based, excitable cells. Following the early work of Winfree and Best, this paper explores the response of a spontaneously spiking Hodgkin-Huxley neuron model to a periodic pulsatile drive. The response as a function of drive period and amplitude is systematically characterized. A wide range of qualitatively distinct responses are found, including entrainment to the input pulse train and persistent chaos. These observations are consistent with a theory of kicked oscillators developed by Qiudong Wang and Lai-Sang Young. In addition to general features predicted by Wang-Young theory, it is found that most combinations of drive period and amplitude lead to entrainment instead of chaos. This preference for entrainment over chaos is explained by the structure of the Hodgkin-Huxley phase resetting curve.
\end{abstract}

\section{Introduction}

The Hodgkin-Huxley model describes action potential generation in certain types of neurons and is a standard model for conductance-based, excitable cells [5, 18, 20]. There is an extensive literature on the response of the Hodgkin-Huxley model to different types of inputs [1, 2, 11, 14, 15, 16, 17, 19, 24, 25], and understanding how single neurons respond to external forcing continues to be relevant for the study of information transmission in neural systems [21, 23]. Because neurons typically communicate via pulsatile synaptic events, it is natural to investigate the response of the Hodgkin-Huxley model to pulsatile inputs. Early studies by Best and Winfree [3, 38] examine the response of a Hodgkin-Huxley model to periodic impulse trains, chracterizing in detail the structure of phase singularities and the transition from degree 1 to degree 0 phase resetting. However, their work does not systematically address the asymptotic dynamical behavior as a function of drive period and amplitude. ${ }^{1}$

This paper studies a spontaneously spiking (i.e. oscillatory) Hodgkin-Huxley neuron model driven by periodic, pulsatile input of fixed amplitude and period, and systematically classifies the response as a function of drive period and amplitude. It is found that:

1. In response to periodic pulsatile forcing of fixed amplitude $A$ and period $T$, a spontaneously spiking Hodgkin-Huxley system can exhibit a wide range of distinct behaviors depending on $A$ and $T$ :

(a) Entrainment: The driven system is stably periodic and its period is a rational multiple of the drive period $T$.

(b) Transient chaos: The system experiences a transient period of exponential instability before entraining to the input. This transient chaos is caused by a Smale horseshoe [13].

\footnotetext{
${ }^{1}$ Takabe, Aihara, and Matsumoto 32 appear to have carried out such a systematic study. But, I was only able to locate an abstract.
} 
(c) Chaos: The system becomes fully chaotic: it possesses a positive Lyapunov exponent and a mixing attractor (see [39] for a review of these concepts).

The response of the pulse-driven neuron is approximately $T_{0}$-periodic in the drive period $T$, where $T_{0}$ is the intrinsic period of the unforced Hodgkin-Huxley oscillator. For example, if the pulse-driven oscillator is chaotic for some drive amplitude $A$ and drive period $T$, then it is likely to be chaotic when driven by a pulse train of amplitude $A$ with period near $T+T_{0}$.

2. The scenarios enumerated above are prevalent in the sense that they correspond to positive-area subsets of the drive period-drive amplitude space. Prevalence, together with the approximate periodicity stated above, imply that each scenario occurs with positive "probability." (See the discussion of Fig. [3] in $\sqrt{3}$ for the precise meaning of probability in this context.) The range of responses and their prevalence are consistent with a theory of nonlinear oscillators developed recently by Qiudong Wang and Lai-Sang Young [33, 34, 35, 36].

3. While chaotic behavior is readily observable, most combinations of drive period and drive amplitude lead to entrainment instead of chaos. This preference for entrainment can be explained by the structure of the phase resetting curve (see 4 of the Hodgkin-Huxley system.

This paper relies heavily on numerical computation and the conceptual framework provided by Winfree's theory of biological rhythms [38] and the work of Q. Wang and L.-S. Young on nonlinear oscillators [34. 35. Phase resetting curves, introduced by Winfree, play a particularly important role here. The phase resetting curve of a nonlinear oscillator is an interval map which captures the asymptotic response of a nonlinear oscillator to a single, pulsatile perturbation. Because they are 1-dimensional objects, phase resetting curves are often easier to understand than the nonlinear oscillators they represent. They are frequently used to infer stable dynamical behavior like phase locking. Wang-Young theory provides a mathematical framework for using phase resetting curves to infer the existence and prevalence of chaotic behavior. Rather than numerically verify the hypotheses of their theorems, we have opted to examine the consequences of the theory directly, relying on a combination of numerical simulation and geometric reasoning to characterize the specific response of the Hodgkin-Huxley model to a periodic pulsatile drive.

For the sake of clarity, parameters are selected to ensure that the Hodgkin-Huxley system possesses a unique limit cycle and no other attracting invariant set. This corresponds to a repeatedly spiking neuron with an unstable rest state. While the scenarios stated above should still hold when the limit cycle coexists with other stable invariant sets, this choice simplifies the interpretation of numerical simulations. Otherwise, a trajectory may jump out of the basin of the limit cycle, which obscures the mechanism described by Wang-Young theory and which Winfree and Best have already investigated thoroughly [3, 38].

The rest of this paper is organized as follows: Section 2 briefly reviews the unforced Hodgkin-Huxley equations and its properties. Main numerical results are summarized in 3 and discussed in $\$ 4$ Section 5 discusses further numerical results, addressing some issues raised in Sections 3 and 4 Section 6 discusses possible extensions and generalizations.

\section{Brief review of the Hodgkin-Huxley model}

The Hodgkin-Huxley equations are a system of nonlinear ordinary differential equations ${ }^{2}$ which describe the way neurons generate spatially and temporally localized electrical pulses [5, 18, 20]. These electrical

\footnotetext{
${ }^{2}$ This paper does not treat the Hodgkin-Huxley PDEs: spatial dependence is not relevant here.
} 
pulses, called action potentials, are the primary way in which neurons transmit information. Action potentials are triggered by sufficiently large membrane voltages, which can be set up by the influx of ions into the cell. A neuron is said to fire or spike when it generates an action potential (Fig. 11. The Hodgkin-Huxley model describes action potential generation in terms of the membrane voltage and dimensionless gating variables which quantify the effective permeability (or conductance) of the membrane for various types of ions.

The original Hodgkin-Huxley equations model action potential generation in the squid giant axon. This giant axon contains two types of membrane ion channels. One type of channel is specific to potassium ions, the other to sodium ions. The state variables of the model are the membrane voltage $v$, the activation $n$ of the potassium channels, and the activation $m$ and inactivation $h$ of the sodium channels. The equations are [18]

$$
\begin{aligned}
\dot{v} & =C^{-1}\left[-I-\bar{g}_{\mathrm{K}} n^{4}\left(v-v_{\mathrm{K}}\right)-\bar{g}_{\mathrm{Na}} m^{3} h\left(v-v_{\mathrm{Na}}\right)-\bar{g}_{\text {leak }}\left(v-v_{\text {leak }}\right)\right] \\
\dot{m} & =\alpha_{\mathrm{m}}(v)(1-m)-\beta_{\mathrm{m}}(v) m \\
\dot{n} & =\alpha_{\mathrm{n}}(v)(1-n)-\beta_{\mathrm{n}}(v) n \\
\dot{h} & =\alpha_{\mathrm{h}}(v)(1-h)-\beta_{\mathrm{h}}(v) h
\end{aligned}
$$

where

$$
\begin{array}{ll}
\alpha_{\mathrm{m}}(v)=\Psi\left(\frac{v+25}{10}\right), & \beta_{\mathrm{m}}(v)=4 \exp (v / 18), \\
\alpha_{\mathrm{n}}(v)=0.1 \Psi\left(\frac{v+10}{10}\right), & \beta_{\mathrm{n}}(v)=0.125 \exp (v / 80), \\
\alpha_{\mathrm{h}}(v)=0.07 \exp (v / 20), & \beta_{\mathrm{h}}(v)=\frac{1}{1+\exp \left(\frac{v+30}{10}\right)}, \\
\Psi(v)=\frac{v}{\exp (v)-1} . &
\end{array}
$$

Each ion channel consists of independent, identical subunits which must all open to allow ions to pass through. The gating variables $m, n$, and $h$ take value in $(0,1)$ and represent the fraction of subunits which are open. The term $n^{4}$ enters into the potassium conductance because potassium channels consist of 4 identical subunits; analogous structures account for the $m^{3} h$ term in the sodium conductance [5]. The gating variable equations are master equations for continuous-time Markov chains with voltage-dependent transition rates $\alpha$ and $\beta$; the Markov chains describe the opening and closing of the corresponding channel subunits. The $\dot{v}$ equation is Kirchoff's current law. Action potentials are downward voltage spikes and a positive I corresponds to an inflow of positively-charged ions. The voltage convention here is that of [18] and opposite contemporary usage: the membrane voltage $v$ is defined by

$$
v=\text { voltage outside }- \text { voltage inside. }
$$

Action potentials are generally initiated by perturbations to the membrane voltage. Such perturbations may be caused, for instance, by the flow of ions across the cell membrane. Because neurons transmit signals through spatially and temporally localized pulses, it is natural to model stimuli as impulses [31]. The simplest type of repetitive, pulsatile stimulus to a neuron is a periodic impulse train. This means replacing the $\dot{v}$ equation above by

$$
\begin{aligned}
\dot{v}= & C^{-1}\left[-I-\bar{g}_{\mathrm{K}} n^{4}\left(v-v_{\mathrm{K}}\right)-\bar{g}_{\mathrm{Na}} m^{3} h\left(v-v_{\mathrm{Na}}\right)-\bar{g}_{\text {leak }}\left(v-v_{\text {leak }}\right)\right] \\
& +A \sum_{k \in \mathbb{Z}} G(t-k T),
\end{aligned}
$$

where $G$ is a "bump" function such that $\int G(t) d t=1$. For simplicity, most of this paper uses the choice $G(t)=\delta(t)$; Section 5.2 discusses the response of the Hodgkin-Huxley system to a pulsatile drive with

$$
G(t)=\left\{\begin{array}{ll}
1 / t_{0}, & 0 \leq t \leq t_{0} \\
0, & \text { otherwise }
\end{array} .\right.
$$




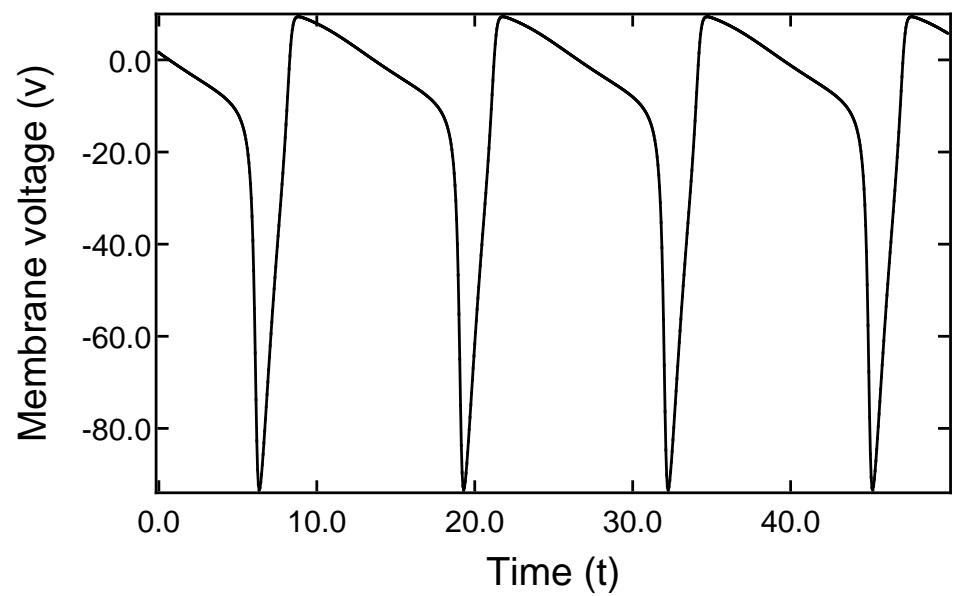

Figure 1: The time course for the Hodgkin-Huxley equations at the parameter values (5). The rapid "spike" followed by a long "recovery" period is typical of the Hodgkin-Huxley equations.

Mathematically, one can also choose to perturb the gating variables, but such perturbations are not entirely natural and are not considered here.

This paper uses the original Hodgkin-Huxley parameters [18]:

$$
\begin{array}{ll}
v_{\mathrm{Na}}=-115 \mathrm{mV}, & \bar{g}_{\mathrm{Na}}=120 \mathrm{~m} \Omega^{-1} / \mathrm{cm}^{2}, \\
v_{\mathrm{K}}=+12 \mathrm{mV}, & \bar{g}_{\mathrm{K}}=36 \mathrm{~m} \Omega^{-1} / \mathrm{cm}^{2}, \\
v_{\text {leak }}=-10.613 \mathrm{mV}, & \bar{g}_{\text {leak }}=0.3 \mathrm{~m} \Omega^{-1} / \mathrm{cm}^{2}, \\
C=1 \mu \mathrm{F} / \mathrm{cm}^{2} . &
\end{array}
$$

Time is measured in milliseconds and current density in $\mu \mathrm{A} / \mathrm{cm}^{2}$.

Figure 2 shows a bifurcation diagram for the unforced Hodgkin-Huxley equations. When $I=0$, the neuron maintains a stable rest state, corresponding to the branch of stable fixed points on the left of the diagram. A sufficiently large value of $I$ causes a neuron to fire repeatedly, which corresponds to the creation of a limit cycle through a saddle-node bifurcation of periodic orbits. Further increasing I destablizes the rest state through a subcritical Hopf bifurcation.

In this paper, the injected current is always set to a value near $I \approx 14$, corresponding to a steady ionic current which destabilizes the rest state. The phenomena studied here are insensitive to the precise value of $I$ as long as it ensures the existence of a stable limit cycle and an unstable fixed point. As explained in the Introduction, these properties simplify the interpretation of numerical simulations. For this choice of $I$, the Jacobian of the Hodgkin-Huxley vector field (Eq. 1) at the unstable fixed point has two real eigenvalues $\{-4.97815,-0.146991\}$ in the left half plane, and a complex conjugate pair $0.0763367 \pm 0.61866 i$ in the right half plane. The fixed point thus has 2-dimensional stable and unstable manifolds. The Lyapunov exponents associated with the limit cycle are $0, \approx-0.20, \approx-2.0$, and $\approx-8.3$. Its period is $T_{0} \approx 12.944$. 


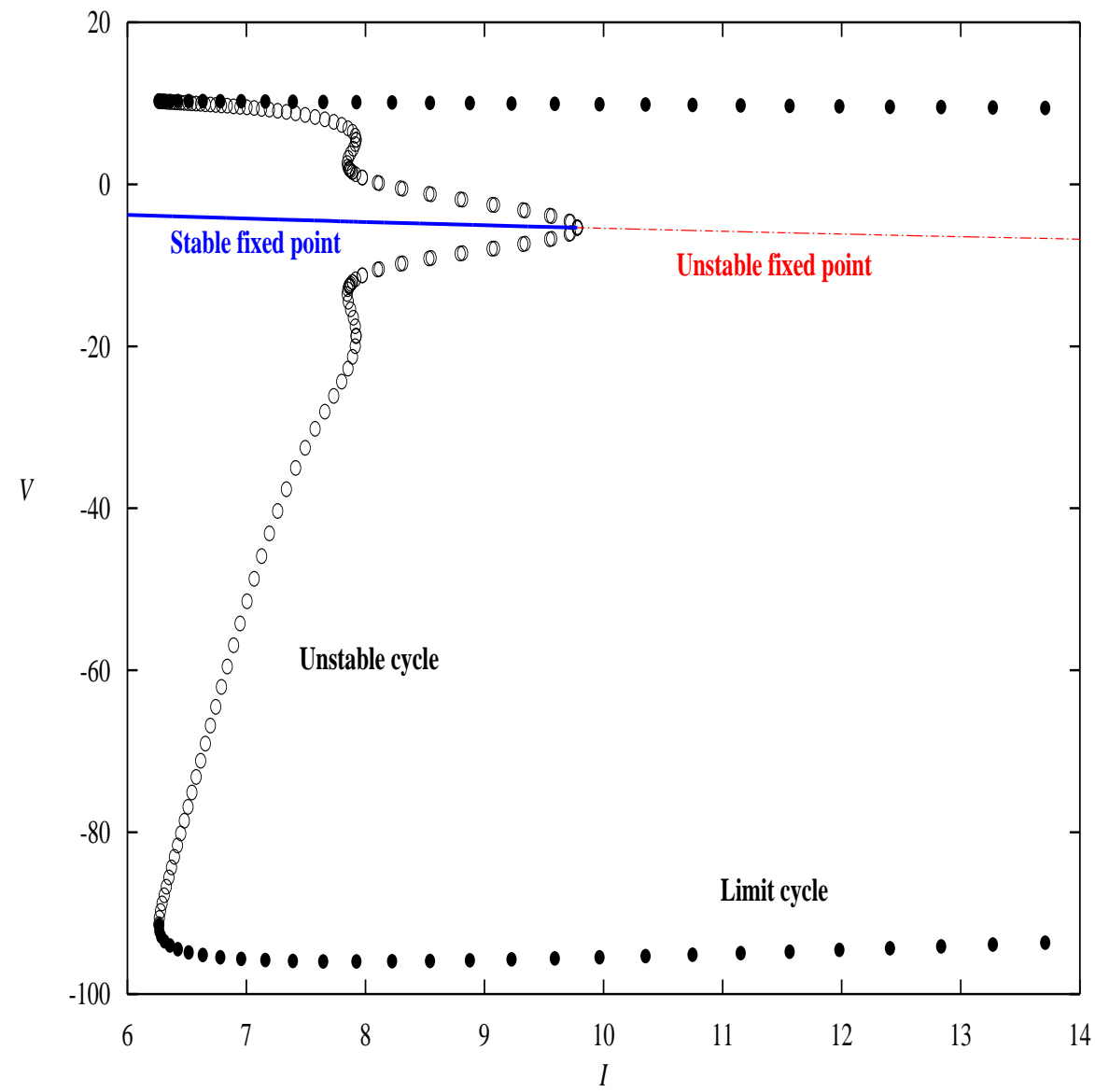

Figure 2: The bifurcation diagram for the Hodgkin-Huxley equations as the injected current $I$ is varied. The line in the middle marks the $v$ coordinate of the rest state. The solid blue part is stable while the dashed red part is unstable. Solid black dots near the top and the bottom of the figure are the maximum and minimum $v$ values of limit cycles. Empty black circles are the maximum and minimum $v$ values of unstable cycles. The fixed point undergoes a subcritical Hopf bifurcation as $I$ increases. This diagram is computed using XPPAUT [7]. 

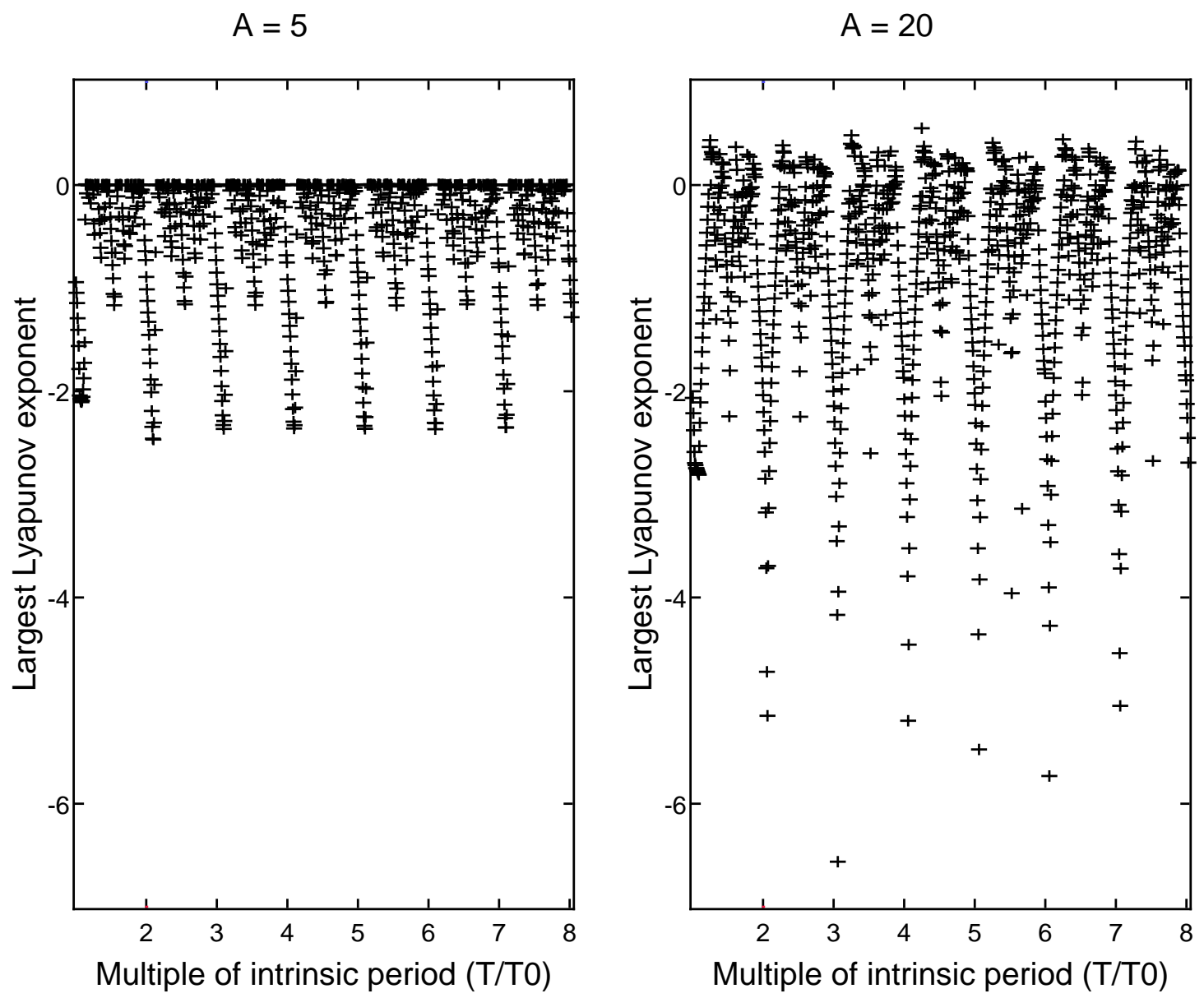

Figure 3: Asymptotic properties of the pulse-driven flow are described by the dynamics of the time- $T$ map $F_{T}$ (see Eq. 6) and its largest Lyapunov exponent $\lambda_{\max }$. Entrainment corresponds to $\lambda_{\max }<0$, and chaos corresponds to $\lambda_{\max }>0$. This figure shows $\lambda_{\max }$ as a function of the drive period $T$, with $T$ ranging from $T_{0} \approx 13$ (the intrinsic period of the unforced Hodgkin-Huxley system; see $₫$ to $8 \cdot T_{0} \approx 101$. Left: Kick amplitude is $A=5$. Right: Kick amplitude is $A=40$. Note (i) $\lambda_{\max }\left(T+T_{0}\right) \approx \lambda_{\max }(T)$; (ii) presence of both positive and negative exponents for strong kicks (right), and only zero and negative exponents for weak kicks (left); and (iii) the presence of more negative exponents than positive ones. See 4 for a discussion. Lyapunov exponents are estimated by iterating $F_{T}$ for 1000 steps and tracking the rate of growth of a tangent vector. 


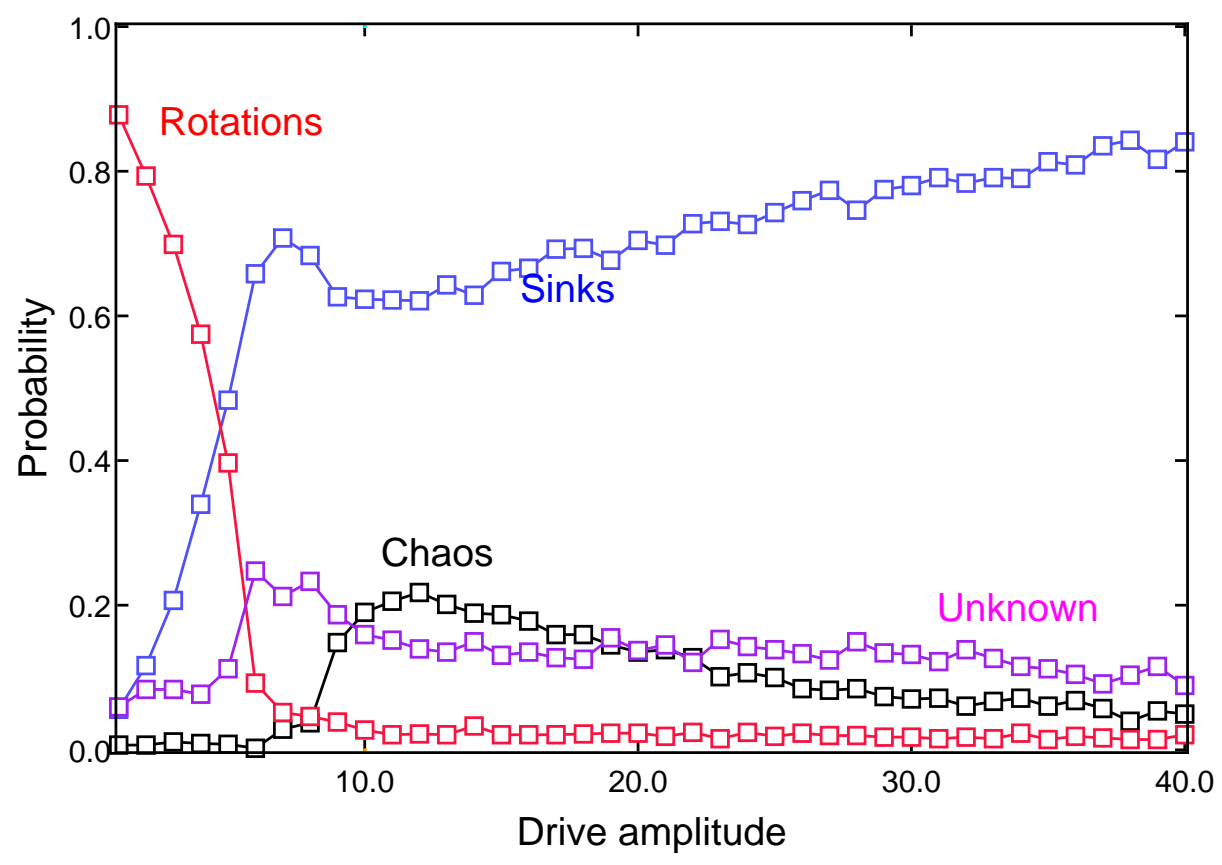

Figure 4: The probability of different response types, as a function of the drive amplitude $A$. For each drive amplitude $A$, the fraction of drive periods $T \in\left[T_{0}, 8 T_{0}\right]$ for which $\lambda_{\max }\left(F_{T}\right)>0$, etc., is computed by sampling from a uniform grid in $\left[T_{0}, 8 T_{0}\right]$. It is natural to equate these fractions with probabilities because the Lyapunov exponents are roughly periodic functions of $T$ (and become more so as $T \rightarrow \infty$ ), as shown in Figure 3 and explained in 4 Empirical definitions: Let $\hat{\lambda}$ denote the estimated Lyapunov exponent and $\epsilon$ the estimated standard error. Then "chaos" is defined as $\hat{\lambda}>3 \epsilon$, "entrainment" $\hat{\lambda}<-3 \epsilon$, and "rotation" $|\hat{\lambda}|<\epsilon / 3$. 


\section{Main numerical results}

Lyapunov exponents provide a convenient way to characterize the asymptotic dynamics of Eq. 3 Let $\phi_{t}: \mathbb{R}^{4} \rightarrow \mathbb{R}^{4}$ denote the flow map generated by the unforced Hodgkin-Huxley equations, $T$ the drive period, and $A$ the drive amplitude. The Poincaré map

$$
F_{T}(v, m, n, h)=\phi_{T}(v+A, m, n, h)
$$

takes a Hodgkin-Huxley state vector $(v, m, n, h)$, applies a pulse of amplitude $A$ to the membrane voltage, then evolves it for time $T$. Iterating the map $F_{T}$ thus gives a stroboscopic record of the state of our pulsedriven Hodgkin-Huxley system before the arrival of each pulse. The long-term dynamical behavior of the pulse-driven Hodgkin-Huxley oscillator can be deduced from the asymptotic dynamics of $F_{T}$, which is characterized by its (largest) Lyapunov exponent $\lambda_{\max }$ [13]:

$$
\begin{array}{ll}
\lambda_{\max }<0 \Leftrightarrow F_{T} \text { has sinks } & \Leftrightarrow \text { kicked flow is entrained to input } \\
\lambda_{\max }=0 \Leftrightarrow F_{T} \text { is quasiperiodic } & \Leftrightarrow \text { kicked flow drifts relative to input } \\
\lambda_{\max }>0 \Leftrightarrow F_{T} \text { chaotic } & \Leftrightarrow \text { kicked flow is chaotic }
\end{array}
$$

(Sinks refer to stable fixed points and stable periodic orbits.) Note that of the scenarios given in the Introduction, transient chaos alone does not appear in this list: Lyapunov exponents, being long-time, average quantities, cannot detect transient chaos.

Figure 3 shows the Lyapunov exponents of $F_{T}$ as a function of $T / T_{0}$, where $T_{0}$ is the period of the Hodgkin-Huxley limit cycle. Different colors correspond to different values of $A$. The periodicity of the response as a function of $T$ is apparent. Because the response type as a function of $T$ is approximately identical over each period $\left[n T_{0},(n+1) T_{0}\right]$, it makes sense to speak of the probability that a randomly chosen drive period $T$ will elicit a particular asymptotic behavior, for example chaos. More precisely, periodicity ensures that the fraction $p_{n}$ of drive periods $T$ in $\left[0, n T_{0}\right]$ for which $\lambda_{\max }>0$ converges to a well-defined limit as $n \rightarrow \infty$. Similar statements hold for $\lambda_{\max }<0$ and $\lambda_{\max }=0$.

Figure 4 shows these probabilities as functions of $A$. At $A=10$, the probability of obtaining a positive exponent is roughly $20 \%$ and the probability of obtaining a negative exponent is roughly $70 \%$. Thus, if one were to pick $T$ randomly out of an interval $\left[N T_{0},(N+1) T_{0}\right]$ for large, fixed integer $N$, the probability that $\lambda_{\max }\left(F_{T}\right)>0$ is about $20 \%$. Figure 4 shows that when $A$ is small, the most likely type of behavior is rotation-like behavior. This possibility becomes less likely as $A$ increases. At the same time, sinks and chaos both become more likely, with sinks dominating the scene. One feature of Figure 4 specific to the pulsedriven Hodgkin-Huxley flow is that when $A$ is large, the system prefers entrainment over chaos in the sense that entrainment has higher probability. This preference is more pronounced as $A$ increases. Note that in computing Lyapunov exponents numerically, we only have access to finite time information. In principle, this means it is virtually impossible to distinguish persistent chaotic behavior from transient chaos caused by a "large" horseshoe (but see 5.1.

In all numerical simulations shown in this paper, Eq. 1 is integrated using an adaptive integrator of Runge-Kutta-Fehlberg type, with an error tolerance of $10^{-6}$ (in the sup norm) [30]. The largest Lyapunov exponent $\lambda_{\max }$ of $F_{T}$ is computed in a straightforward manner, by choosing a nonzero unit vector $w \in \mathbb{R}^{4}$ and estimating the rate of growth of $\left\|\left(D F_{T}\right)^{n} w\right\|$. The matrix-vector product $\left(D F_{T}\right)^{n} w$ is easily computed via the variational equations $\dot{x}=H(x), \dot{w}=D H(x) \cdot w$ for the Hodgkin-Huxley vector field $H$ ( $D H$ is the Jacobian matrix of $H$; see [10] for details). 


\section{Discussion}

\subsection{Response to a single pulse: phase resetting curves}

This section reviews phase resetting curves. See Winfree [38], Glass and Mackey [11], and Brown, Moehlis, and Holmes [4] for more details and applications, and Guckenheimer and Holmes [13] for background information on dynamical systems theory. See [7, 8, 9, 37] for further discussions of phase resetting curves.

Let $\phi_{t}: \mathbb{R}^{n} \rightarrow \mathbb{R}^{n}$ be a flow generated by a smooth vector field with a hyperbolic limit cycle $\gamma$. Such a limit cycle represents a stable nonlinear oscillator. The basin of attraction of $\gamma$ is denoted $B(\gamma)$. The hyperbolicity of $\gamma$ guarantees that points in $B(\gamma)$ converge to $\gamma$ exponentially fast. (It is convenient to use $\gamma$ to refer to both the trajectory $\gamma: \mathbb{R} \rightarrow \mathbb{R}^{n}$ and the invariant point set it defines.) An impulsive perturbation ("kick") to the nonlinear oscillator can be defined by specifying a kick amplitude $A$ and a kick direction $\hat{K}: \mathbb{R}^{n} \rightarrow \mathbb{R}^{n}$ and defining a family of kick maps

$$
K_{A}(x)=x+A \cdot \hat{K}(x),
$$

so that kicks send each point $x \in \mathbb{R}^{n}$ to $K_{A}(x)$. For what follows, $K_{A}$ should be smooth and satisfy $K_{A}(B(\gamma)) \subset B(\gamma)$.

The Hodgkin-Huxley system with the value of $I$ given in 2 is a nonlinear oscillator whose basin $B(\gamma)$ is an open subset of $\mathbb{R}^{4}$. The kick map corresponding to an instantaneous voltage spike is simply $K_{A}(v, m, n, h)=(v+A, m, n, h)$. As in $₫$ it is convenient to introduce the time- $T$ map

$$
F_{T}=\phi_{T} \circ K_{A},
$$

where $\circ$ denotes function composition. Iterating $F_{T}$ gives a stroboscopic record of the system state before the arrival of each kick, and thus describes the long-time dynamics of the flow $\phi_{t}$ under repeated, $T$-periodic kicks.

Because the phase dimension $n$ may be large, the dynamics of $F_{T}: \mathbb{R}^{n} \rightarrow \mathbb{R}^{n}$ may be difficult to analyze. Winfree observed that every point near the limit cycle $\gamma$ must converge to $\gamma$ as $t \rightarrow \infty$, so the flow near $\gamma$ is dominated by the rotational motion along $\gamma$. Thus, one can reduce the dimension of the phase space from $n$ to 1 , at least heuristically. To do this, first define the phase function $\theta: \gamma \rightarrow\left[0, T_{0}\right)$ by fixing a reference point $x_{0} \in \gamma$ and requiring that $\phi_{\theta(x)}\left(x_{0}\right)=x$ for all $x \in \gamma$. By construction, $\theta$ satisfies $\frac{d}{d t}(\theta(\gamma(t)))=1,0 \leq$ $t<T_{0}$. The function $\theta$ can be extended to a function $\theta: B(\gamma) \rightarrow\left[0, T_{0}\right)$ by projecting along strong-stable manifolds ${ }^{3}$ : if $y$ is a point in the basin of $\gamma$ then $\theta(y)$ is defined to be $\theta(x)$, where $x$ is the unique point such that $y \in W_{\mathrm{ss}}(x)$. This definition of phase preserves the property that $\frac{d}{d t}\left(\theta\left(\phi_{t}(x)\right)\right)=1$.

Consider the limit [12]

$$
\bar{F}_{T}=\lim _{n \rightarrow+\infty} F_{T+n T_{0}} .
$$

The map $\bar{F}_{T}$ is well-defined on the basin of $\gamma$ and retracts the basin onto $\gamma$, i.e. $\bar{F}_{T}(x) \in \gamma$ for all $x \in B(\gamma)$. Thus, $\bar{F}_{T}$ induces an interval map $f_{T}:\left[0, T_{0}\right) \rightarrow\left[0, T_{0}\right)$ which, given the current phase of the system, yields the new phase after kicking and evolving the system for time $T$. That is, $f_{T}(\theta(x))=\theta\left(F_{T}(x)\right)$ for all $x \in B(\gamma)$.

\footnotetext{
${ }^{3}$ The strong-stable manifold $W_{\mathrm{ss}}(x)$ of $x \in \gamma$ is the set

$$
W_{\mathrm{ss}}(x)=\left\{y \in \mathbb{R}^{n}: \lim _{n \in \mathbb{Z}, n \rightarrow+\infty} \phi_{n T_{0}}(y) \rightarrow x\right\} .
$$

When the vector field generating $\phi_{t}$ is smooth, the strong-stable manifolds are (locally) smooth submanifolds of $\mathbb{R}^{n}$. The strong-stable linear subspace $E_{\mathrm{ss}}(x)$ is the tangent space of $W_{\mathrm{ss}}(x)$ at $x$. See [12]13.
} 


\begin{tabular}{|c|c|c|}
\hline Drive amplitude $A$ & Prob. of sink near plateau & Prob. of $\lambda_{\max }<0$ \\
\hline 5 & $41 \%$ & $48 \%$ \\
10 & $58 \%$ & $62 \%$ \\
20 & $68 \%$ & $70 \%$ \\
30 & $76 \%$ & $78 \%$ \\
\hline
\end{tabular}

Table 1: Estimates of the probability of obtaining sinks near the plateau, as a function of $A$. The data for this table is computed by trying about 40 values of $T$ for each choice of $A$ and examining the graph of the first return map to the interval $[4,10]$ (chosen to coincide with the "plateau") and its intersection(s) with the diagonal.

The map $f_{T}$ is the phase resetting curve ${ }^{4}$, or more precisely the finite phase resetting curve (infinitesimal phase resetting curves [4, 7] are not needed here). By construction, it has the property that

$$
f_{T+\delta}(t)=f_{T}(t)+\delta\left(\bmod T_{0}\right)
$$

Thus, the family of maps $\left\{f_{T}\right\}$ is periodic in $T$.

Periodicity in drive period $T$. The approximate periodicity of $\lambda_{\max }\left(F_{T}\right)$ seen in Figure 3 is easy to understand heuristically: kicking the oscillator every $T$ seconds and kicking it every $T+T_{0}$ seconds should yield the same asymptotic response because the oscillator simply traverses $\gamma$ at frequency $1 / T_{0}$ between kicks. One can restate this using phase resetting curves: if the drive period $T$ is sufficiently large and $\theta\left(x_{0}\right)=t_{0}$, then the $f_{T}$-orbit $\left(t_{0}, f_{T}\left(t_{0}\right), f_{T}^{2}\left(t_{0}\right), \ldots\right)$ should closely follow the phases $\left(\theta\left(x_{0}\right), \theta\left(F_{T}\left(x_{0}\right)\right), \theta\left(F_{T}^{2}\left(x_{0}\right)\right), \ldots\right)$ of the corresponding $F_{T}$-orbit. Since $f_{T+T_{0}}=f_{T}$, this suggests that $\lambda_{\max }\left(F_{T+T_{0}}\right) \approx \lambda_{\max }\left(F_{T}\right)$.

Preference for entrainment. Figure [5] shows phase resetting curves for the Hodgkin-Huxley equations for various values of drive period $T$ and drive amplitude $A$. For sufficientlly small values of $A$, the phase resetting curves are circle diffeomorphisms: either there are sinks (i.e. stable fixed pionts or stable periodic orbits), or the map is conjugate to a rotation on a circle and the response of the kicked oscillator drifts relative to the periodic drive. As $A$ increases, the graph of $f_{T}$ rather quickly folds over and acquires critical points. A striking feature of the graphs in Figure 5 is the "plateau," a phase interval over which $f_{T}$ varies very slowly. Another striking feature is the "kink" around $\theta \approx 9.8$. These features are discussed in more depth in $\$ 5$ For now, notice that the plateau provides a simple mechanism for creating sinks: changing the kick period $T$ shifts the graph of $f_{T}$ vertically. Whenever the graph intersects the diagonal with a derivative $\left|f_{T}^{\prime}\right|<1$, then a stable fixed point is created.

This mechanism can be used to verify the results of Figure 4 compute the graph of the first return map of $f_{T}$ to an interval around the plateau, then shift the graph vertically using a number of different values of $T$ and estimate the fraction of $T^{\prime}$ s for which $f_{T}$ has a stable fixed point (see Figure 6 . Table 1 shows the results. For $A=10$, the $58 \%$ probability of sinks corresponds fairly closely with Figure 4 It is unclear whether the ambiguous exponents in Figure 4 really represent positive or negative Lyapunov exponents. If a significant fraction of the ambiguous exponents are really negative, then they must come from small sinks.

\footnotetext{
${ }^{4}$ Wang and Young refer to phase resetting curves as singular limits. Phase resetting curves are also sometimes called phase transition curves 11 .
} 


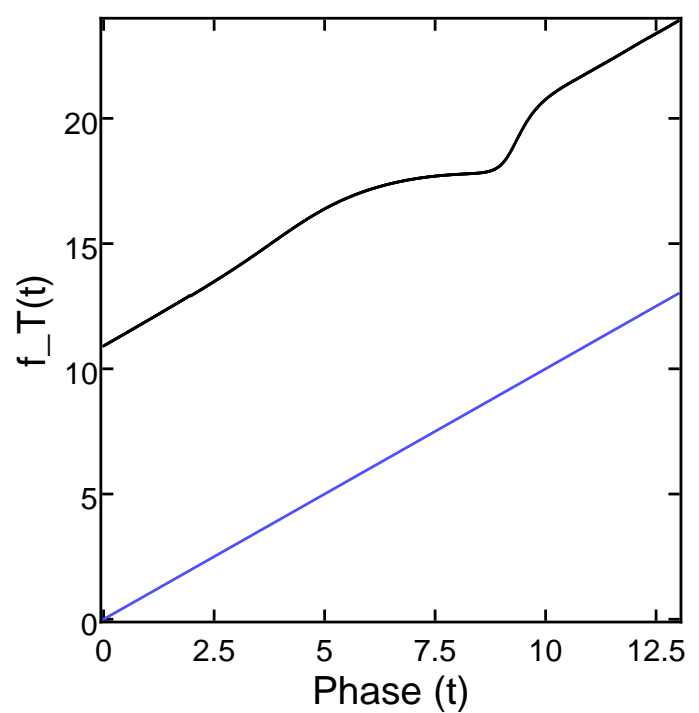

(a) $A=5$

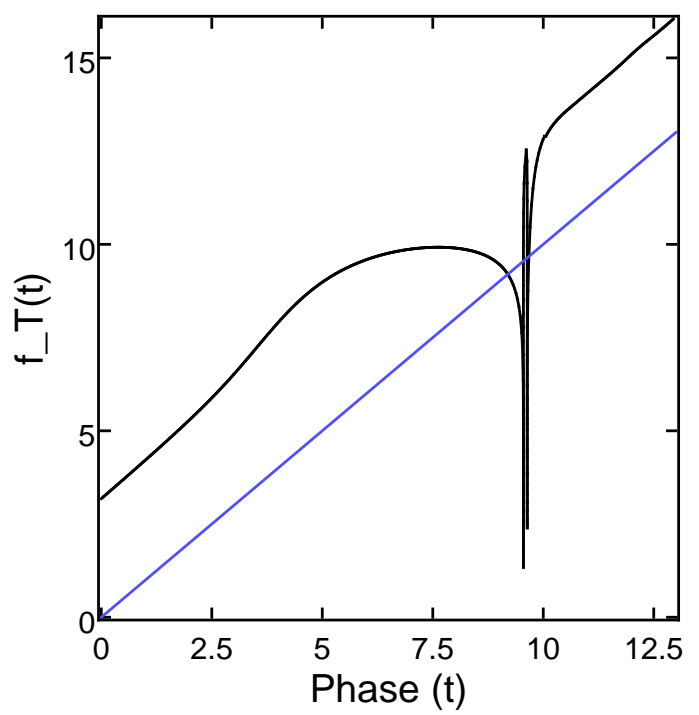

(b) $A=10$

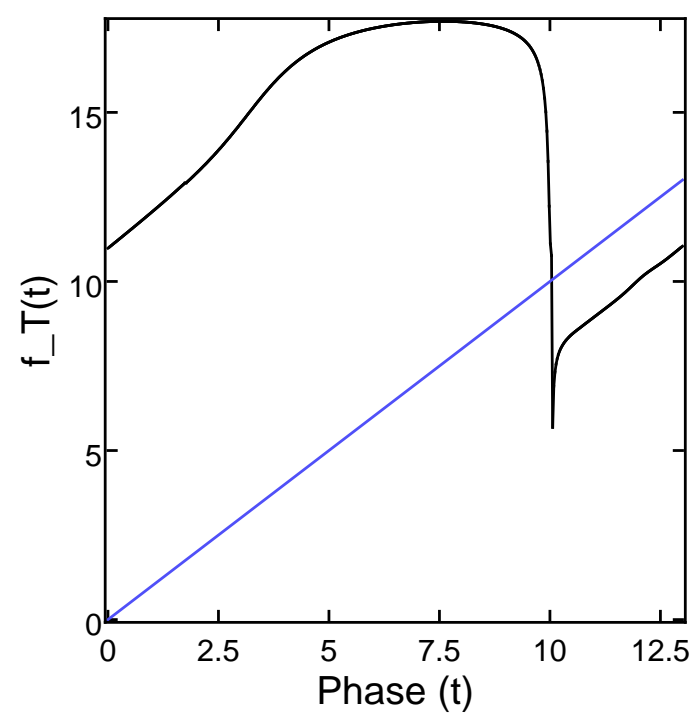

(c) $A=20$

Figure 5: The graph of the lift $\tilde{f}_{T}$ of $f_{T}$, i.e. the unique continuous map $\mathbb{R} \rightarrow \mathbb{R}$ such that $\tilde{f}_{T}=f_{T}$ on $\left[0, T_{0}\right)$ and $\tilde{f}_{T}\left(t+T_{0}\right)=\tilde{f}_{T}(t)\left(\bmod T_{0}\right)$, for the pulse-driven Hodgkin-Huxley equations. Drive amplitudes are (a) $A=5$, (b) $A=10$, and (c) $A=20$. The precise value of the drive period $T$ is not so important; varying $T$ shifts the graph vertically (Eq. 11). Note that $f_{T}$ has winding number 1 for $A=5$ and $A=10$, and has winding number 0 for $A=20$. The numerical data suggests that the degree changes around $A \approx 13.589$; the precise geometric mechanism is not clear. Note that phase ranges from 0 to the intrinsic period $T_{0} \approx 12.9$ of the Hodgkin-Huxley limit cycle. 


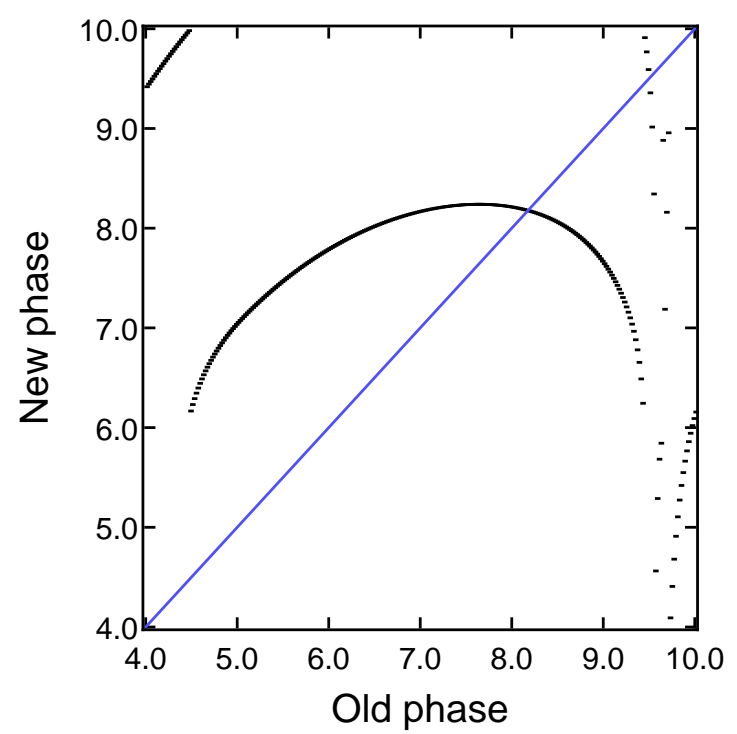

Figure 6: The first return map $R_{f_{T}}$ to the interval $[4,10]$ (chosen to enclose the plateau), for $A=10$ and $T=17.6$. The blue line marks the diagonal.

Note on numerics. Phase resetting curves are computed here using a variation of the Ermentrout-Kopell adjoint method [4, 8]. The method is described in the Appendix. A systematic comparison of this method to existing methods for computing phase resetting curves is beyond the scope of the present paper and will be presented elsewhere.

\subsection{Response to repeated pulses: Wang-Young theory}

Phase resetting curves provide simple, intuitive explanations for many dynamical properties of pulsedriven nonlinear oscillators. For our spiking Hodgkin-Huxley oscillator, explicitly-computed phase resetting curves show why our pulse-driven neuron prefers entrainment over chaos. In order to infer asymptotic behavior, there needs to be a correspondence between the orbits of $f_{T}$ and $F_{T}$, and the phase of $x \in B(\gamma)$ generally does not determine the phase of $F_{T}(x)$ : it may only do so approximately for a finite number of iterates. When $\lambda_{\max }\left(f_{T}\right)<0$, this is enough to show that $f_{T}$ orbits indeed approximate the phases of $F_{T}$. Inferring chaotic behavior for $F_{T}$ from $f_{T}$ is far more difficult. Wang-Young theory provides a mathematical framework for inferring chaotic behavior using phase resetting curves, and in addition explains why chaotic phenomena (and all the other scenarios) is prevalent.

Shear is an important ingredient of Wang-Young theory. Let $\gamma$ be the limit cycle which represents the unforced nonlinear oscillator. Near $\gamma$, the dynamics follows the periodic, rotational motion on $\gamma$. Shear refers to the presence of an angular velocity gradient around $\gamma$ : the stronger the shear, the sharper the angular velocity changes at $\gamma$. In two dimensions, this means the flow runs much faster on one side of $\gamma$ than on the other; in the presence of strong shear, strong stable manifolds tend to become more nearly tangent to $\gamma$.

Shear and its interaction with kicks is illustrated in a simple model in Figure 7 In the presence of strong shear, most ways of kicking the oscillator which take advantage of shear (e.g. kicks which do not take $x \in \gamma$ too close to the strong-stable manifold $W_{\mathrm{ss}}(x)$ ) will cause segments of the limit cycle to stretch and fold as 


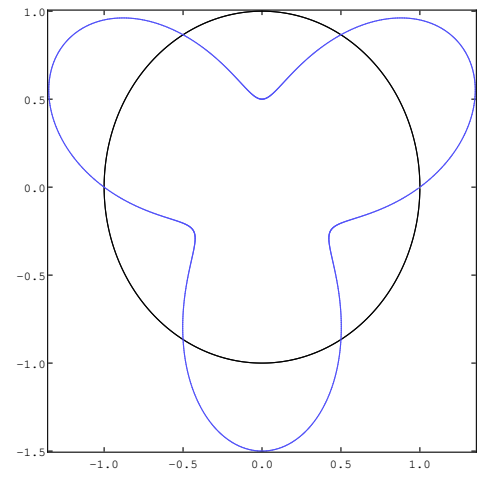

(a)

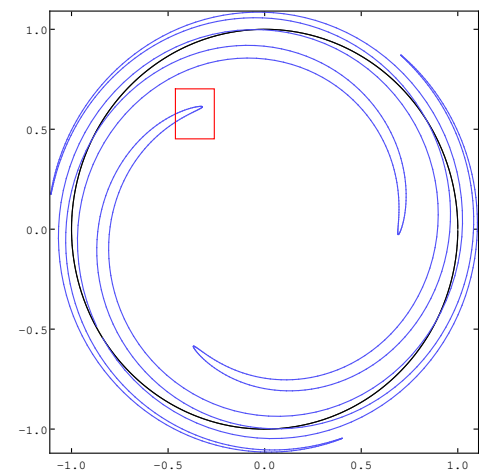

(b)

Figure 7: A simple model (see Eq.12) which illustrates the effect of shear. In (a), a large number of initial conditions are placed around a limit cycle (the black circle) and a radial kick is applied to each point. The blue curve shows the resulting positions of each initial condition. In (b), the kicked points are allowed to flow. The red box contains one of the turning points (see 4.2.

they fall back toward $\gamma$. The phase space stretching caused by shear manifests itself in phase intervals over which $\left|f_{T}^{\prime}\right|>1$. This expansion is conducive to chaotic behavior. However, shear also creates region of contraction around "turning points," an example of which is highlighted in Figure 7 Such turning points correspond to critical points on the phase resetting curve and can easily counteract the expansion needed for a positive Lyapunov exponent. The competition between expansion and contraction is the main source of difficulty in proving $\lambda_{\max }\left(F_{T}\right)>0$.

To infer the existence of parameters for which the time- $T$ map $F_{T}$ is fully chaotic, Wang and Young use results from their previous work on strange attractors with 1 expanding direction and a roughly toroidal geometry [33, 36]. Their main result gives conditions under which there must be $T$ for which $\lambda_{\max }\left(F_{T}\right)>0$. Furthermore, one can find such "chaotic parameters" near "nice" values of $T$ for which $f_{T}$ has a positive Lyapunov expoent. Applying the general theory to kicked oscillators requires checking certain geometric conditions. This has been done for a few concrete classes of models [27, 34, 35]. To illustrate the consequences of the theorems, consider the simple mechanical system [34]

$$
\ddot{\theta}(t)+\lambda \dot{\theta}(t)=\mu+A \cdot \hat{K}(\theta(t)) \sum_{n \in \mathbb{Z}} \delta(t-n T) .
$$

This model was first studied by Zaslavsky, who discovered that this simple system can exhibit fully chaotic behavior [40]. For Eq. 12] it can be shown that the full range of scenarios enumerated in the Introduction take place and that they are all prevalent. More precisely, Wang and Young prove (see Theorems 1-3 in [34]) that:

1. Invariant curve \& weak kicks: When the drive amplitude $A$ is sufficiently small (which is equivalent to having a large enough contraction rate $\lambda$ ), there exists a simple closed curve $\tilde{\gamma}$ to which all orbits of $F_{T}$ converge and which is invariant under $F_{T}$. Moreover, we have the following dichotomy:

(a) Quasiperiodic attractors: There exist a set of $T$ of positive Lebesgue measure for which $F_{T}$ is topologically conjugate to an irrational rotation. In this case, $F_{T}$ is uniquely ergodic on $\tilde{\gamma}$. 
(b) Gradient-like dynamics: There exists an open set of $T$ such that $F_{T}$ has a finite number of periodic sinks and saddles on $\tilde{\gamma}$, and every orbit converges to one of these periodic orbits.

2. Gradient-like dynamics without an invariant curve: As $A$ increases (or $\lambda$ decreases), the invariant curve $\tilde{\gamma}$ breaks up. Nevertheless, there continues to be an invariant set (no longer a simple closed curve) on which gradient-like dynamics persists.

3. Transient chaos: For even larger $A$ or smaller $\lambda$, Smale horseshoes (see [13]) will form. Horseshoes can coexist with sinks and saddles, creating transient chaos.

4. Chaos: In the presence of sufficiently strong shear, there exists a positive measure set of drive periods $T$ for which $F_{T}$ is fully chaotic in the sense that it possesses (i) a strange attractor with a positive Lyapunov exponent; (ii) at least 1 and at most finitely many ergodic SRB measures ${ }^{5}$ with no zero Lyapunov exponents; (iii) a central limit theorem; (iv) exponential decay of correlations if a power $F_{T}^{N}$ is mixing for some SRB measure $v$.

Note that this list of (fairly well-understood) scenarios may not be exhaustive. Other scenarios or combinations of scenarios are not excluded by the theory. Also, the kicks in Eq.12 are purely radial. This is not strictly necessary; any kick map which takes advantage of shear will do. See [34] for precise conditions and proofs.

\section{Further results}

\subsection{More on the Hodgkin-Huxley phase resetting curve}

\section{Plateau}

The plateau in the phase resetting curve for our pulse-driven Hodgkin-Huxley model (see Figure 5) corresponds a segment $\bar{\gamma}$ of the limit cycle $\gamma$ which becomes nearly parallel to a strong-stable manifold after receiving a kick. This can be seen by examining the factors which contribute to the derivative $f_{T}^{\prime}$ and which can potentially cause $f_{T}^{\prime}$ to become small over a relatively large phase interval. This can be checked by writing $f_{T}$ as a composition of other functions and differentiating.

Let $\gamma: \mathbb{R} \rightarrow \mathbb{R}^{4}$ denote the limit cycle trajectory. If we choose $\gamma(0)$ so that $\theta(\gamma(0))=0$, then $\theta(\gamma(t))=t$ for all $t \in\left[0, T_{0}\right)$, and

$$
\begin{aligned}
f_{T} & =\theta \circ F_{T} \circ \gamma \\
& =\theta \circ \phi_{T} \circ K_{A} \circ \gamma .
\end{aligned}
$$

Changing $T$ does not affect $f_{T}^{\prime}$, so we can set $T=0$. Let $f=f_{0}$. Then $f=\theta \circ K_{A} \circ \gamma$ and the chain rule gives

$$
f^{\prime}=\left(D \theta \circ K_{A} \circ \gamma\right) \cdot\left(D K_{A} \circ \gamma\right) \cdot \dot{\gamma}
$$

But $K_{A}(v, m, n, h)=(v+A, m, n, h)$, so its Jacobian $D K_{A}$ is the identity matrix, and for all $t \in\left[0, T_{0}\right)$,

$$
\begin{aligned}
f^{\prime}(t) & =D \theta\left(K_{A}(\gamma(t))\right) \cdot \dot{\gamma}(t) \\
& =\left|D \theta\left(K_{A}(\gamma(t))\right)\right| \cdot|\dot{\gamma}(t)| \cdot \cos \left(\operatorname{angle}\left(D \theta\left(K_{A}(\gamma(t))\right), \dot{\gamma}(t)\right)\right) \\
& =\left|D \theta\left(K_{A}(\gamma(t))\right)\right| \cdot|\dot{\gamma}(t)| \cdot \sin (\measuredangle(t)),
\end{aligned}
$$

\footnotetext{
${ }^{5}$ SRB measures are natural invariant measures for dissipative dynamical systems. They characterize the asymptotic behavior of a Lebesgue-positive measure set of initial conditions and have a number of nice mathematical properties. See Young [39 for an introduction.
} 
where $\measuredangle(t)$ is the angle between $\dot{\gamma}(t)$ and the strong-stable manifold at $K_{A}(\gamma(t))$. The last step uses the fact that the phase function $\theta: B(\gamma) \rightarrow\left[0, T_{0}\right)$ is constant on strong-stable manifolds (see 4 . This implies that the gradient $D \theta(x)$ is everywhere orthogonal to $W_{\mathrm{ss}}\left(x^{*}\right)$, where $x^{*}$ is the unique point in $\gamma$ having the same phase as $x$. The factors in Eq. 14 thus have simple, geometric meaning: $\gamma(t)$ is a point on the limit cycle $\gamma$ and $|\dot{\gamma}(t)|$ is the speed of the limit cycle at that point; $\measuredangle(t)$ is the angle between $\dot{\gamma}(t)$ and the strong-stable manifold at $K_{A}(\gamma(t))$; and $|D \theta|$ measures the rate at which the phase is changing at $K_{A}(\gamma(t))$.

Figure 8 shows $f^{\prime}$ alongside the 3 factors in Eq. 14 The figure shows that the plateau, where $f_{T}^{\prime}$ becomes nearly 0 over a long phase interval, coincides with the near-vanishing of $\measuredangle(t)$. The other factors of $f^{\prime}$ stay nearly constant over this interval. Thus, there is a segment $\bar{\gamma}$ of the limit cycle $\gamma$, corresponding to the phase interval where $\measuredangle(t)$ is small, such that $K_{A}(\bar{\gamma})$ is nearly tangent to a strong-stable manifold. That the segment $\bar{\gamma}$, which may be small as a subset of $\mathbb{R}^{4}$, corresponds to a large phase interval, is due to the relatively slow speed of the limit cycle near $\bar{\gamma}$.

What this argument does not explain is the robustness of this tangency (equivalently, the robustness of the plateau) as the drive amplitude $A$ increases (see Fig. [5]. This requires a detailed analysis of the geometry (in $\mathbb{R}^{4}$ !) of the strong-stable manifolds (see $₫$.

Numerical computation of $D \theta$. Figure 8 requires the numerical computation of the gradient $D \theta(x)$ for $x \in B(\gamma)$. This can be done as follows:

Fix $x \in B(\gamma)$ and consider $\phi_{T_{0}}^{n}(x)$. Clearly, the $\operatorname{limit}_{n \rightarrow \infty} \lim _{T_{0}}^{n}(x)=x^{*}$ exists and has the property that $x^{*} \in \gamma, \theta\left(x^{*}\right)=\theta(x)$, and $x \in W_{\mathrm{ss}}\left(x^{*}\right)$. Set $\pi_{\mathrm{ss}}(x)=x^{*}$. Then $\pi_{\mathrm{ss}}$ projects $B(\gamma)$ onto $\gamma$ and is the identity map on $\gamma$. Furthermore, the nullspace of the Jacobian matrix $D \pi_{\mathrm{ss}}(x)$ of $\pi_{\mathrm{ss}}$ is the tangent to $W_{\mathrm{ss}}\left(\pi_{\mathrm{ss}}(x)\right)$ at $x$, by construction.

To compute $D \theta(x)$, the foregoing discussion suggests that we compute $D \phi_{T_{0}}^{n}(x)$ for some large finite $n$. For any finite $n$, the singular values of $D \phi_{T_{0}}^{n}(x)$ consist of a dominant singular value $\sigma_{1}$ and 3 nearly zero singular values $\sigma_{2}, \sigma_{3}$, and $\sigma_{4}$. The $\sigma_{i} \rightarrow 0$ as $n \rightarrow \infty$ for $i=2,3,4$. Denote the left and right singular vectors associated with $\sigma_{1}$ by $u$ and $v$. It is easy to check that the right eigenvector $v$ is orthogonal to the null space of $D \pi_{\mathrm{ss}}(x)$ and hence tangent to $D \theta$.

This computation requires a relatively accurate estimate of the intrinsic period $T_{0}$ of the limit cycle $\gamma$, without which the computation would not converge. This paper adopts the following strategy: instead of estimating $T_{0}$ just once and reusing its value, solve the system of 24 equations

$$
\dot{x}_{1}=H\left(x_{1}\right), \dot{x}_{2}=H\left(x_{2}\right), \dot{J}=D H\left(x_{2}\right) \cdot J
$$

with initial conditions

$$
x_{1}(0) \in \gamma, x_{2}(0)=x, J(0)=\operatorname{Id}_{4 \times 4}
$$

where $H$ is the Hodgkin-Huxley flow field and $x$ is the point at which we would like to evaluate $D \theta$. Note that $x_{1}, x_{2} \in \mathbb{R}^{4}$ and $J \in \mathbb{R}^{4 \times 4}$. The solution of these equations then give $x_{2}(t)=\phi_{t}(x)$ and $J(t)=$ $D \phi_{t}(x)$. The reference trajectory $x_{1}$ is only used to count the number of periods which have elapsed, and the trajectory $\left(x_{2}, J\right)$ is used to compute $D \pi_{\mathrm{ss}}(x)$. This procedure works fairly well in practice.

\section{Kink}

It is natural to ask whether $f_{T}$ (see Fig. 5), for $A=10$ or $A=20$, is discontinuous around the kink. A discontinuity indicates that there are points in a neighborhood of $\gamma$ which can be kicked outside the basin of $\gamma$. This is not likely the case: Figure 9 shows a magnified view of the phase resetting curve near the kink; the graph does not include any numerical interpolation. The figure is obtained by fixing a small 


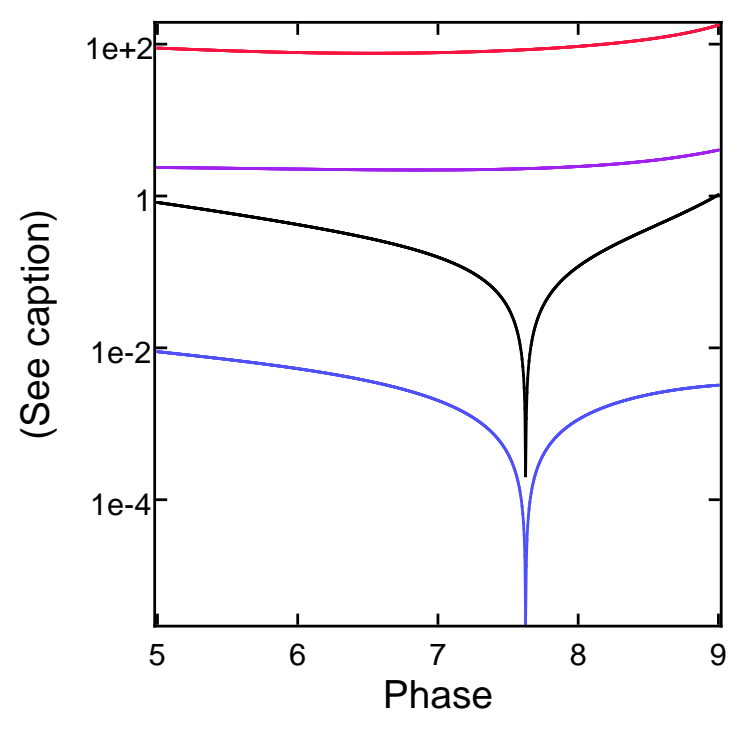

(a)

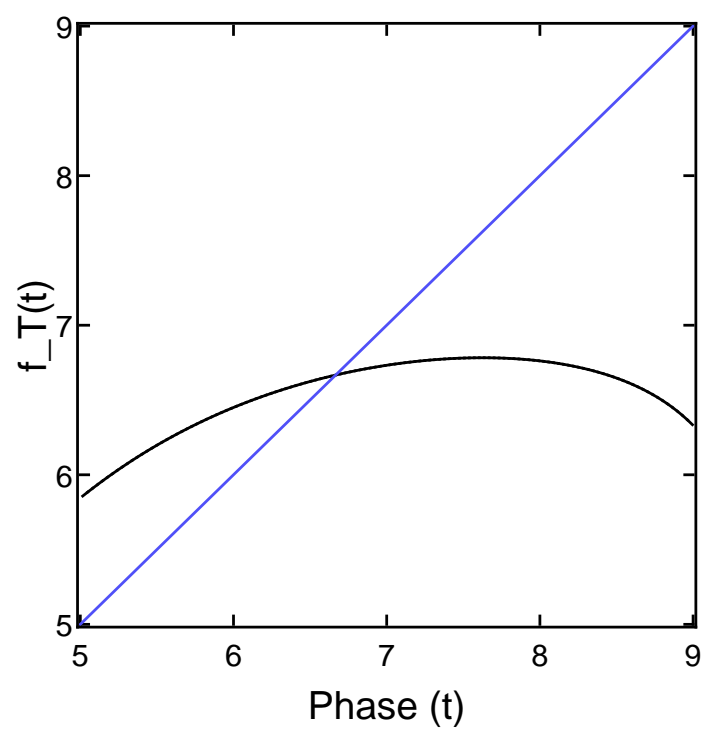

(b)

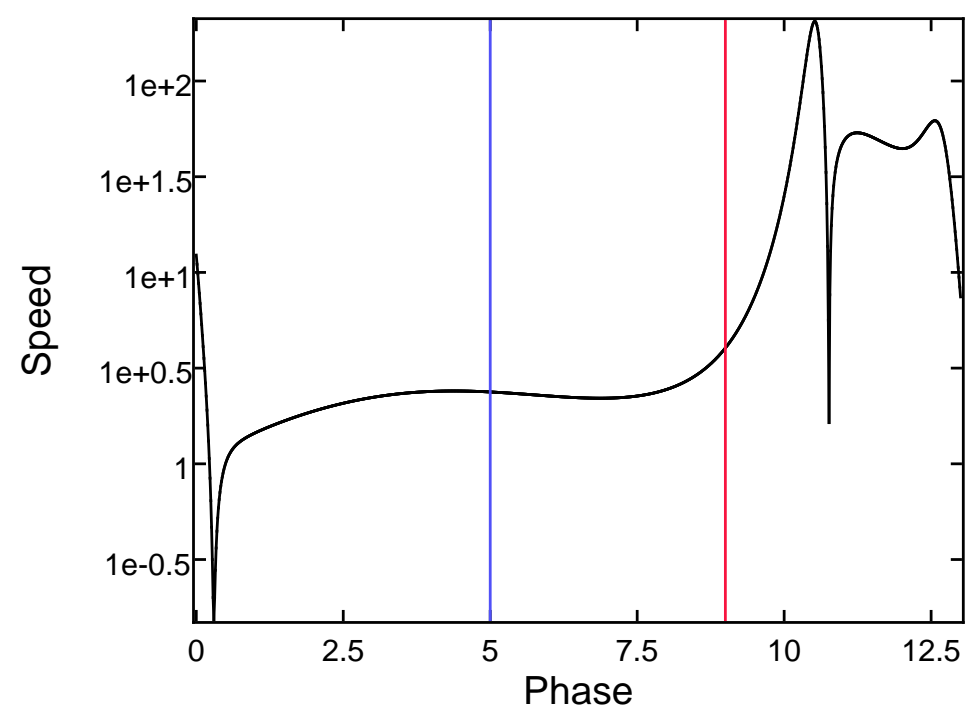

(c)

Figure 8: The origin of the plateau: in (a), the curves are (i) Black: $\left|f^{\prime}(t)\right|$; (ii) Blue: $|\sin (\measuredangle(t))|$; (iii) Red: $\left|D \theta\left(K_{A}(t)\right)\right|$; (iv) Purple: $|\dot{\gamma}(t)|$. In (b), the graph of $f_{T}$ near the plateau is shown for reference. Here, $A=10$. (c) The speed of the Hodgkin-Huxley flow along $\gamma$. The vertical lines mark the interval $[5,9]$, which is part of the plateau. 


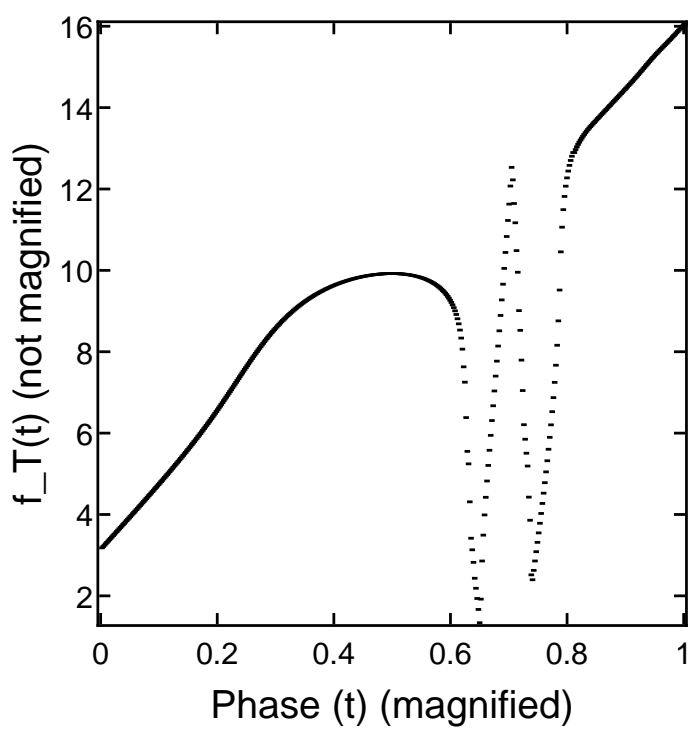

(a)

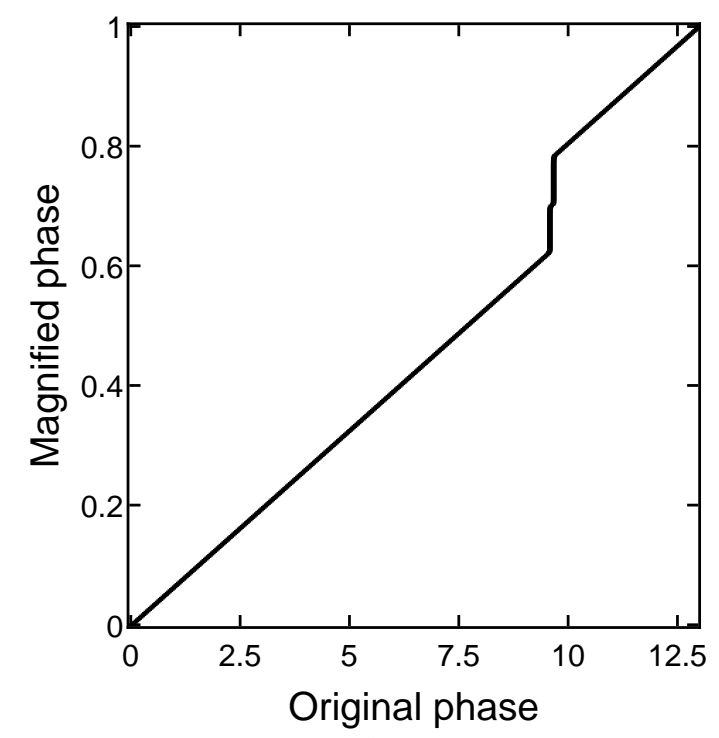

(b)

Figure 9: (a) The graph of $f_{T} \circ g^{-1}$ with drive amplitude $A=10$, with the abscissa shown in a new coordinate system $\theta^{\prime}=g(\theta)$ to magnify the region around the "kink." No interpolation is done in this figure: only actually computed points are shown. (b) The graph of the coordinate transformation $g$. The map $g$ is generated automatically by the simple adaptive algorithm described in the appendix.

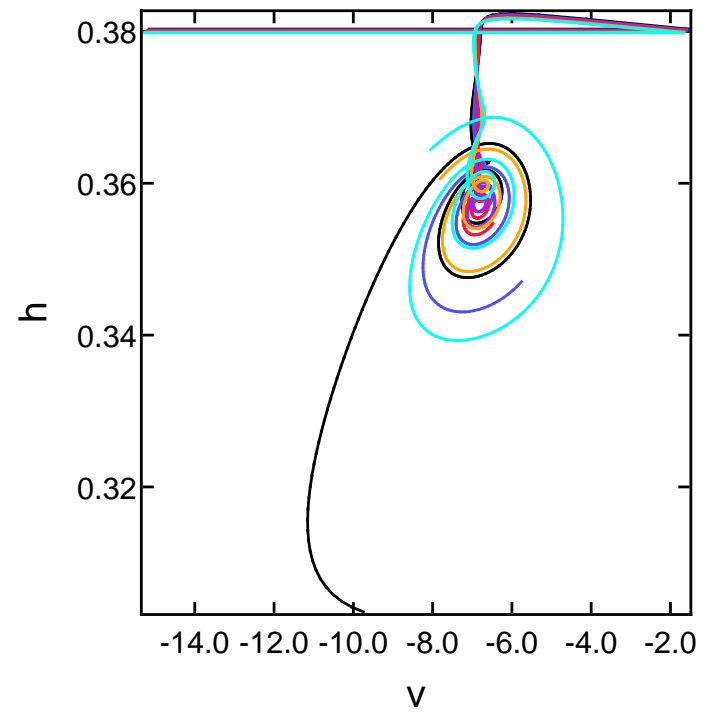

(a)

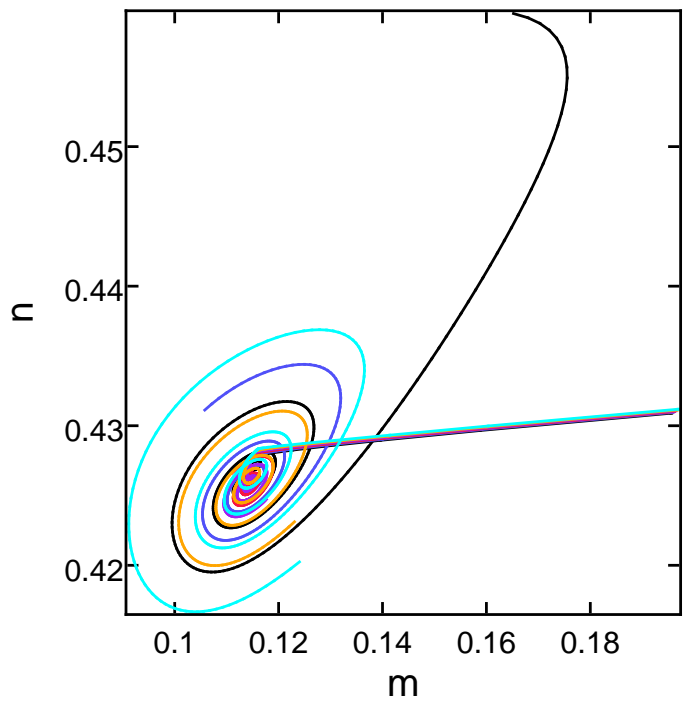

(b)

Figure 10: A suggestive picture: in (a), a segment of $\gamma$, starting in the upper left corner of the picture, is kicked straight across to the upper right corner. It then follows the flow toward the fixed point for some time before spiraling away. The overall direction of motion is top to bottom. (b) Another view of the approach to the fixed point. The overall direction of motion here is right to left. The kick amplitude is $A=13.589$. 


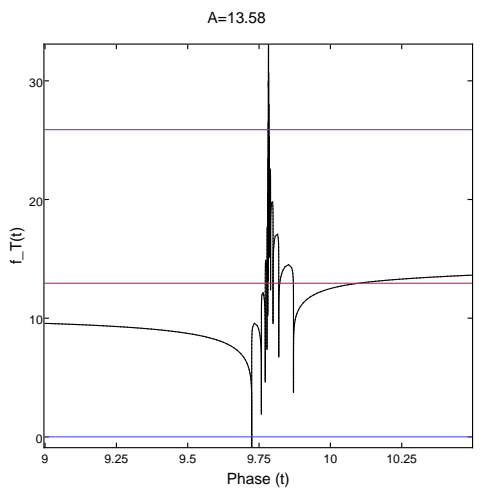

(a)

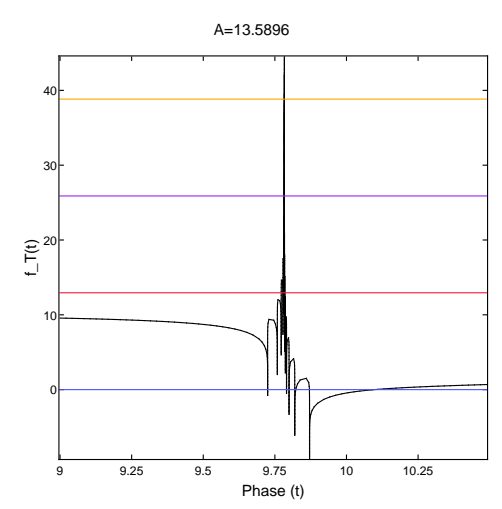

(d)

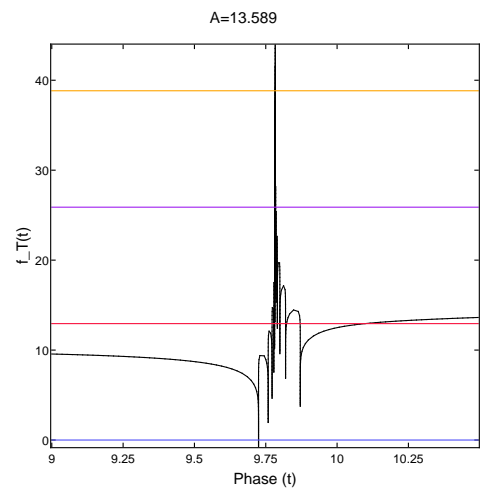

(b)

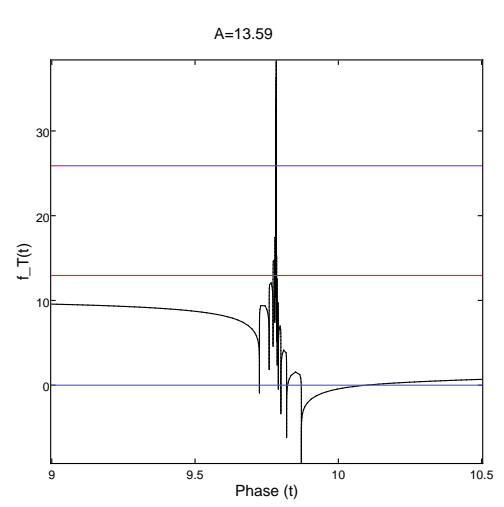

(e)

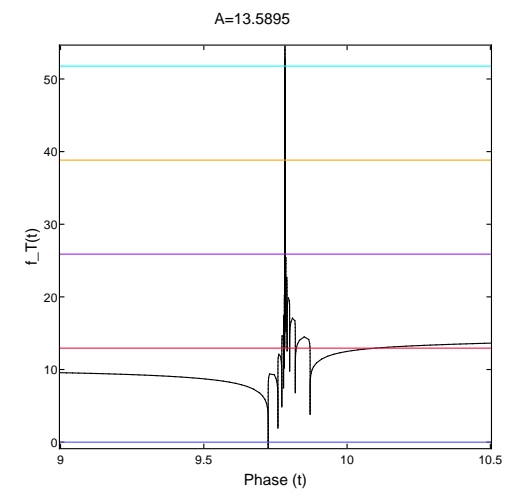

(c)

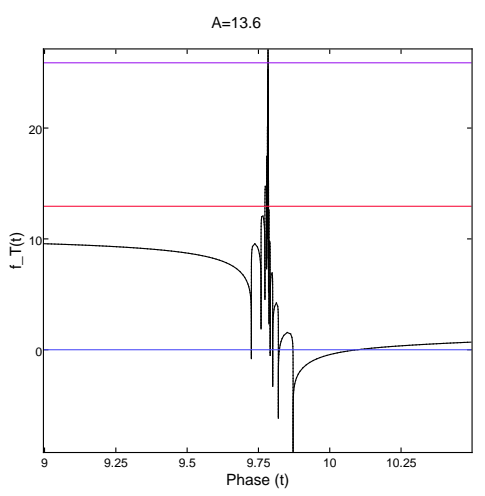

(f)

Figure 11: Graphs of $f_{T}$ for kick amplitudes $A$ approaching $A_{\text {crit }}$ from below (a-c) and above (d-f). The horizontal lines mark integral multiples of $T_{0}$. 
parameter $\delta>0$ and adaptively refining the grid $\left\{\theta_{n}\right\}$ on which the phase resetting curve is evaluated until $\left|f_{T}\left(\theta_{n+1}\right)-f_{T}\left(\theta_{n}\right)\right| \leq \delta$. In Figure $9 \delta$ is set to 0.1 . The adaptive procedure (see the Appendix) continues to converge for smaller values of $\delta$.

Figure 10 suggests an explanation for the kink: that it is likely caused by a segment of $\gamma$ being kicked near the stable manifold of the unstable fixed point. This would cause the segment to wind around the stable manifold and eventually spirals away from the fixed point. (Recall that the two unstable eigenvalues of the fixed point form a complex conjugate pair.) In the process the kicked segment spreads apart and its subsets pick up different amounts of time delays. However, because the Hodgkin-Huxley phase space is 4-dimensional, Figure 10 cannot give a reliable picture of the dynamics: projecting onto 2 dimensions loses too much information.

The scenario sketched above predicts that there exist a critical kick amplitude $A_{\text {crit }}$ at which $K_{A}(\gamma)$ intersects the stable manifold of the fixed point. (There may be more than 1 intersection, and more than 1 value of $A$ which cause intersections.) As $A \rightarrow A_{\text {crit }}$ the phase resetting curve should start winding around $S^{1}$ more and more. This can be numerically tested: an estimate of $A_{\text {crit }}$ is computed using the Nelder-Meade algorithm [30] to minimize the closest distance of a trajectory to the fixed point. This yields a critical value $A_{\text {crit }} \approx 13.58953 \ldots$. When $A=A_{\text {crit }}$ exactly, $f_{T}$ should wind around infinitely many times and possess a singularity near the location(s) of intersection. For $A \neq A_{\text {crit }}, f_{T}$ remains smooth, but as $A \rightarrow A_{\text {crit }}, f_{T}$ should develop a singularity and blow up. See Figure 11

\section{Horseshoes \& transient chaos}

Wang-Young theory also guarantees the existence of $T^{\prime}$ 's for which $F_{T}$ exhibits transient chaos, i.e. $F_{T}$ possesses a Smale horseshoe [13] together with a sink. The coexistence of a horseshoe with a sink has the following effect on the dynamics: almost every $F_{T}$-orbit would eventually fall into a sink, but an orbit which wanders near a horseshoe would dance around unpredictably for a finite number of iterations. Two nearby orbits which enter the vicinity of a horseshoe can emerge widely separated, and fall into the sink out of phase (unless the sink happens to be a fixed point). In terms of time series data, this kind of behavior can be recognized by looking at pairs of trajectories and finding that they chaotically "flutter" about before settling down into a steady periodic motion, likely out of phase.

In contrast to entrainment and chaos, transient chaotic behavior is difficult to observe in the pulsedriven Hodgkin-Huxley system. This is because the most likely place to find a horseshoe is near the kink, where the expansion so strong that most trajectories escape very quickly. Nevertheless, it is possible to find indirect evidence for horseshoes in the pulse-driven Hodgkin-Huxley model. To do so, one looks for an interval $I \subset\left[0, T_{0}\right)$ such that $f_{T}(I)$ gets mapped completely across $I$ at least twice. It is easy, for example, to find a "small" horseshoe around the kink in the phase resetting curve. See Figure12 The phase interval $I$ tells us the rough location of a horseshoe for $F_{T}$.

To go from such an interval $I$ to a horseshoe for the full map $F_{T}$, it is necessary to (i) blow up the corresponding segment of $\gamma$ to form an open set $U \subset \mathbb{R}^{4}$ such that $F_{T}(U)$ intersects $U$ at least twice, and the intersection stretches all the way across $U$ in the unstable direction (along $\gamma$ ); and (ii) find invariant cones [13]. This can be done in a straightforward manner and is not discussed further here.

\subsection{Miscellaney}

\section{Decay of correlations}

Wang-Young theory predicts that when the dynamics of a pulse-driven oscillator is chaotic and there is a unique SRB measure, time correlations (more precisely time autocovariance functions) decay exponentially 


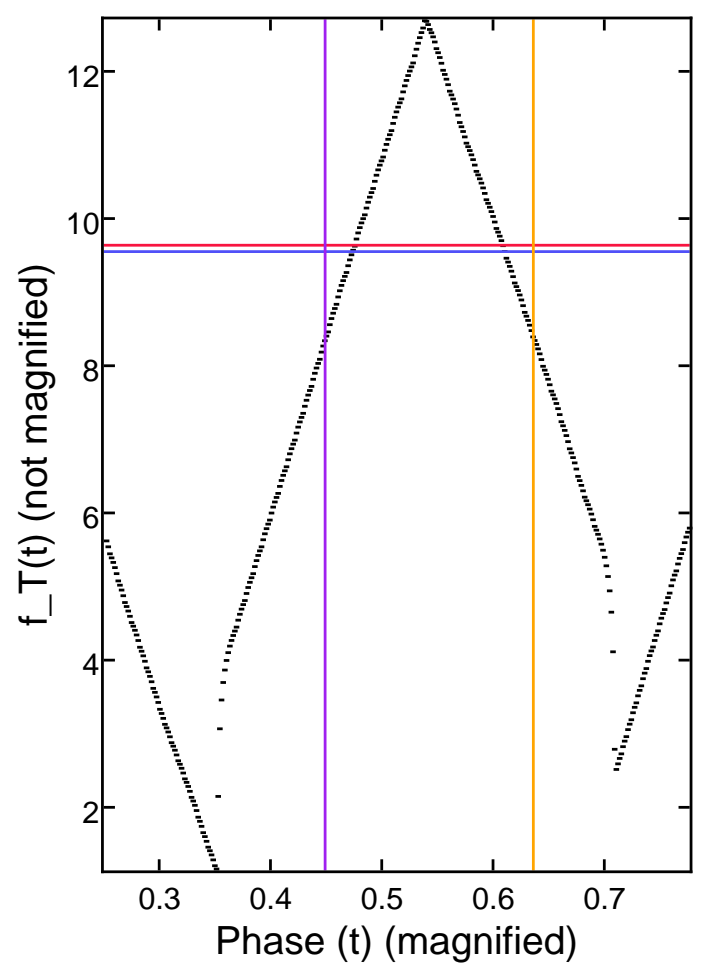

Figure 12: The phase resetting map and an interval $I$ (marked by the straight lines) which maps across itself twice. The abscissa (but not the ordinate) is shown in transformed coordinates. Here, $A=10$ and $T=81$. This is a "small" horseshoe: because the derivative of $f_{T}$ is so large there (on the order of $10^{3} \sim 10^{4}$ ), most numerically computed orbits escape the horseshoe after a few iterates. 


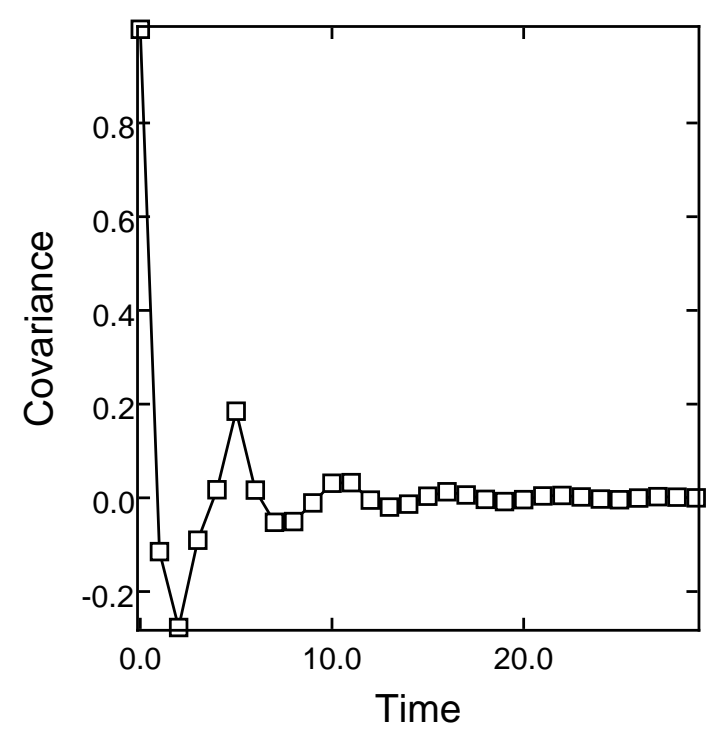

(a) Linear

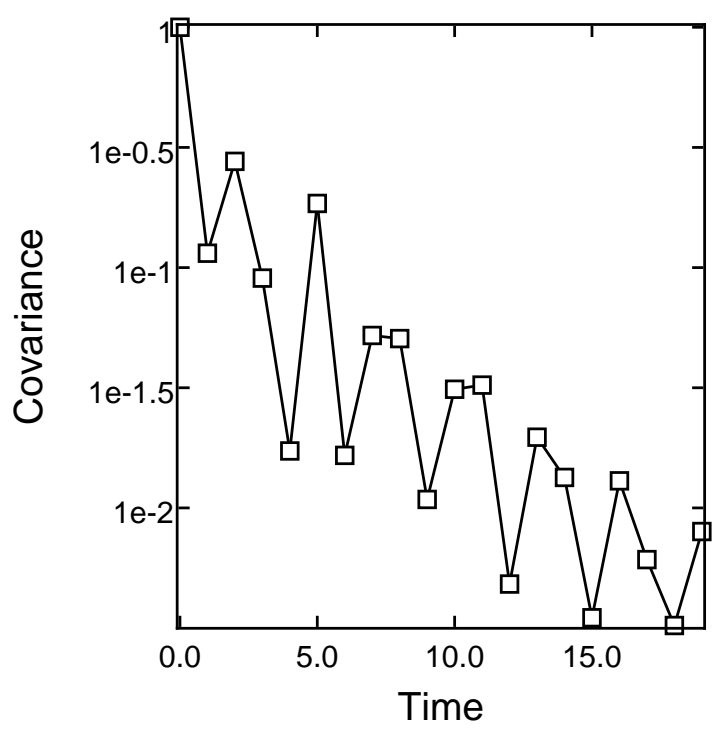

(b) Semilog

Figure 13: Normalized autocorrelation function $C_{v v}(n)$ for the $v$ variable of the time- $T$ map $F_{T}$. This graph indicates that the dynamics is mixing, but the data is insufficient to confirm that the system is exponentially mixing.

fast in time. Figure 13 shows the time autocovariance function

$$
C_{v v}(n)=\int\left(v \circ F_{T}^{n}\right) \cdot v d \mu-\left(\int v d \mu\right)^{2}
$$

for the voltage variable $v$ ( $\mu$ is an ergodic invariant measure). While it clearly decays as $n \rightarrow \infty$ and thus provides evidence that the invariant measure is mixing, the data is not sufficient to confirm that the decay is exponential.

\section{Response to finite-duration pulses}

A natural variation on the numerical experiments of previous sections is to replace instantaneous impulses with finite-duration pulses. Heuristically, if the pulse durection $t_{0}$ is less than the fastest of the intrinsic timescales of $\gamma$, the resulting response should be essentially the same as the response to instantaneous impulses. With $I \approx 14$, these time scales are 12.944 (= the period), 5.1, 0.50, and 0.12 (corresponding to the negative Lyapunov exponents).

Figure 14 summarizes the numerical results for $t_{0}=0.05$ (shorter than all time scales), 0.3 (shorter than all but one time scale), 2.75 (shorter than all but two fastest time scales), and 9.0 (very slow, not really pulsatile in any sense of the word). These graphs should be compared to Figure 4 The pulse amplitude is adjusted so that the total amount of charge delivered is the same as an impulse of amplitude $A$. Interestingly enough, the behavior seen earlier are quite robust and disappear only when $t_{0}=9$. These results suggest that the contracting directions do not mix very much over $\gamma$, and only the slowest contracting time scale participates in the production of chaotic behavior. 


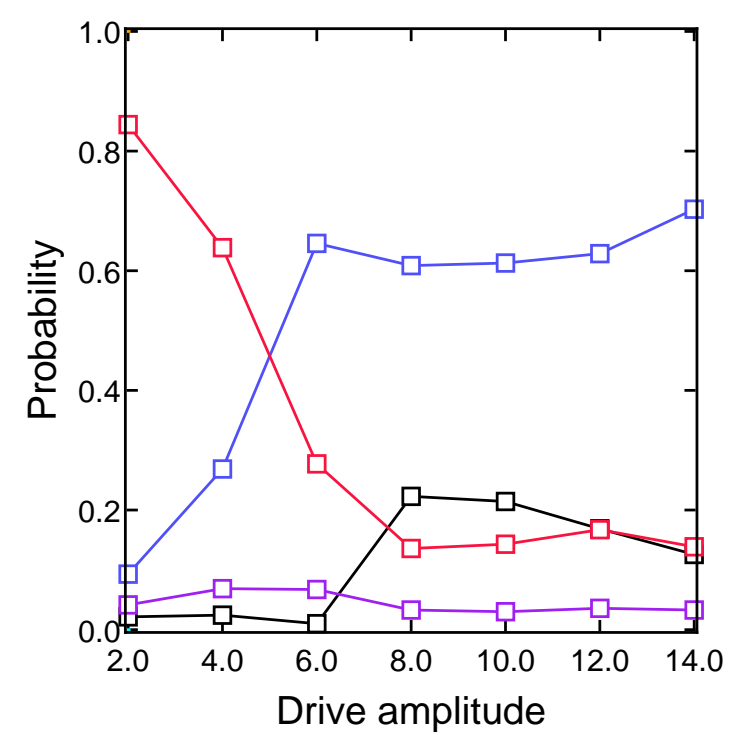

(a)

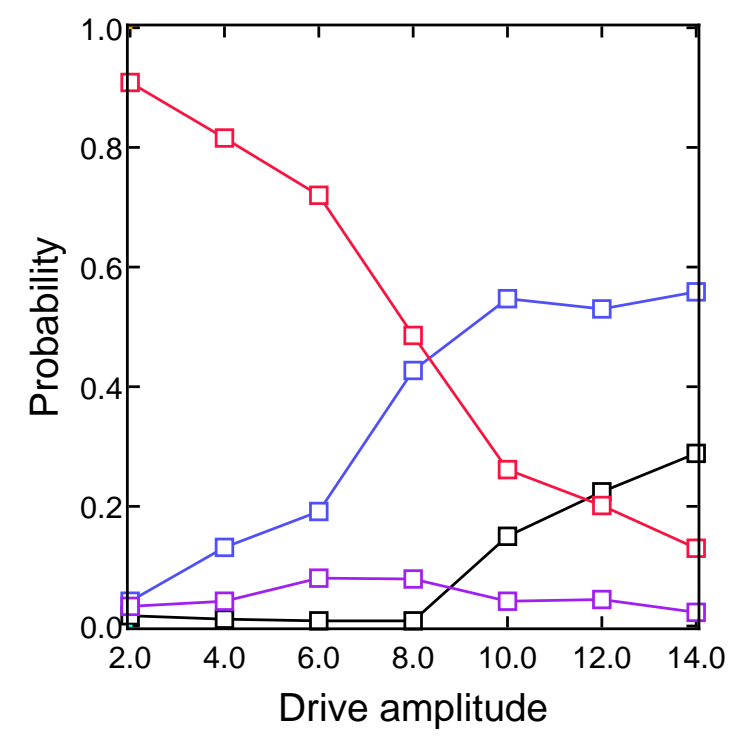

(c)

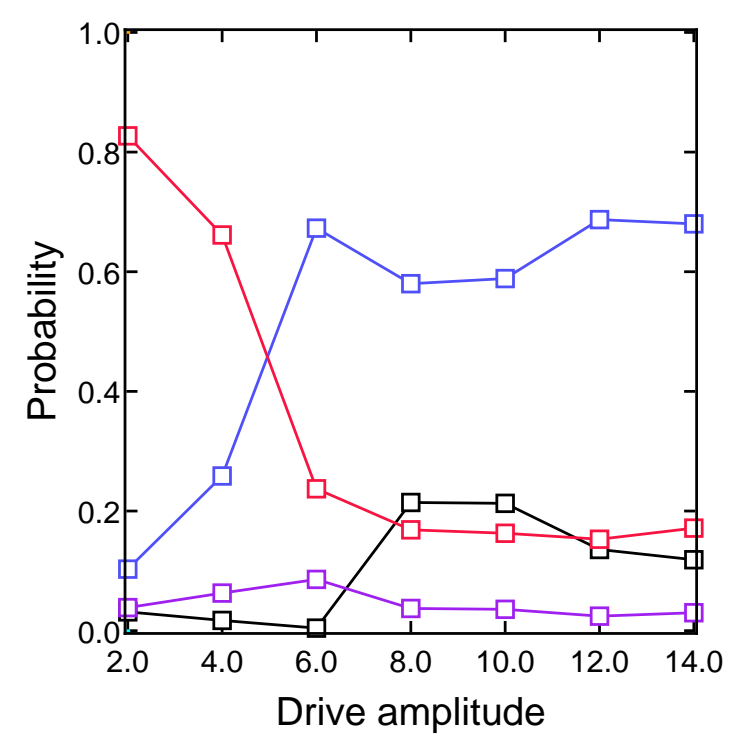

(b)

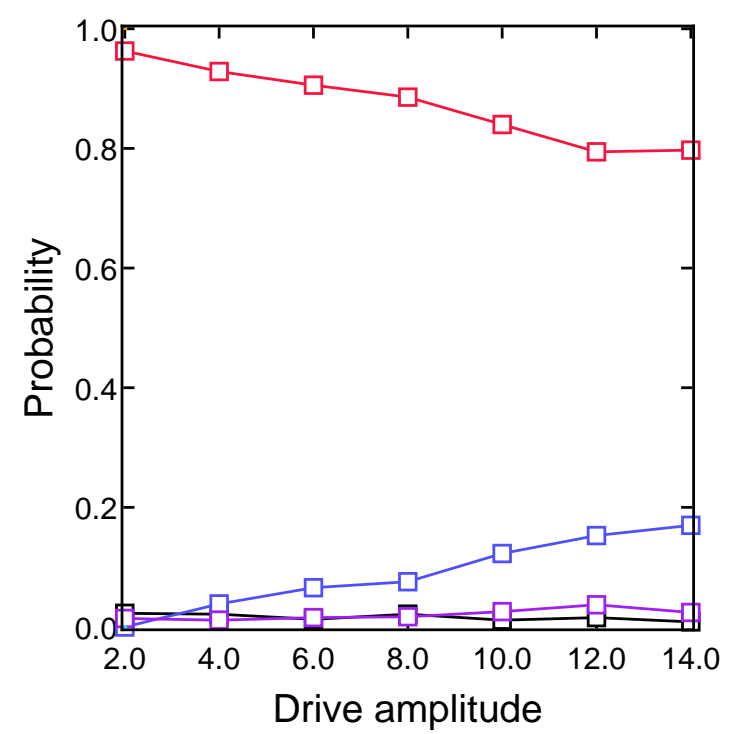

(d)

Figure 14: Distribution of Lyapunov exponents for $I \approx 14$ with finite-duration pulse of duration (a) $t_{0}=$ 0.05 , (b) $t_{0}=0.3$, (c) $t_{0}=2.75$, and (d) $t_{0}=9.0$. See Figure 4 caption for details. Recall that the Lyapunov exponents of the unperturbed limit cycle $\gamma$ are $-0.196,-2.01$, and -8.31 , corresponding to relaxation times of $5.1,0.50$, and 0.12 . 


\section{Outlook}

The results reported here show that the pulse-driven Hodgkin-Huxley model (3) responds to low-frequency (relative to the intrinsic period $T_{0}$ of the spiking neuron) periodic impulse forcing in a wide range of ways. Depending on the drive period and drive amplitude, the response can range from entrainment to fully chaotic behavior. This is consistent with the predictions of Wang-Young theory. Furthermore, as shown in 4 it is possible to explain some phenomena specific to our pulse-driven Hodgkin-Huxley oscillator in terms of special features of the phase resetting curve and provide a partial understanding of the source of these features.

There are some interesting directions for future work:

Random kick times. The shape of the Hodgkin-Huxley phase resetting curve suggests that if one were to drive a Hodgkin-Huxley neuron using a pulse train with random kick times, the resulting random dynamical system can have a negative Lyapunov exponent. This is because the phase resetting curve moves up and down from kick to kick, and for any kick time distribution which is sufficiently uniform (e.g. an exponential distribution), the probability that the plateau intersects the diagonal is high. The size of the plateau suggests that over many iterates, contraction may dominate expansion, leading to a negative Lyapunov exponent. A negative Lyapunov exponent implies that two Hodgkin-Huxley neurons, when driven by a common pulse train with random kick times, will synchronize. That is, the plateau provides a way to create a "random fixed point" [22]. These predictions are consistent with preliminary numerical results and with a perturbation theory developed by Nakao et. al. for randomly-kicked oscillators in the limit of weak kicks [26]. The heuristic, geometric argument sketched above may lead to an extension of their result to the regime of strong kicks.

This synchronization mechanism has also been studied numerically by Doi in the context of a simple pieceise linear map [6]. In addition, there is an extensive literature on noise-induced synchrony in neural models, including white-noise-driven Hodgkin-Huxley equations [29. 41]. Models exhibiting noiseinduced synchrony provide a concrete framework for exploring neural reliability [21, 23].

Robustness of the phase resetting curve. How robust are the features (plateau, kink) of the HodgkinHuxley phase resetting curve under perturbations to parameters? How robust is the geometry of the neartangency of kicked segments and strong-stable manifolds responsible for forming the plateau? As the range of phenomena predicted by Wang-Young theory may be present in two-dimensional models like the Morris-Lecar or FitzHugh-Nagumo, these models may provide a good starting point for exploring these questions in lower-dimensional settings.

\section{A Appendix: Computation of phase resetting curves}

All numerical calculations of phase resetting curves reported in this paper use the algorithm described here. It is closely related to an algorithm due to Ermentrout and Kopell [8]. It is presented here for the sake of completeness; a systematic comparison with existing methods will appear elsewhere. The algorithm computes the phase resetting curve for finite-size perturbations but can be adapted to compute phase resetting curves for infinitesimal perturbations. See Appendix A in [4] and [28, 37]) for more general discussions of phase resetting curves, and Ermentrout, Pascal, and Gutkin [9] for a discussion of computing phase resetting curves experimentally. 
The basic idea is to numerically compute the strong-stable linear subspaces along the limit cycle. Then, using these linear subspaces as approximations to strong-stable submanifolds, project a kicked point down to the limit cycle, where the phase can be estimated. The algorithm really computes strong-stable linear subspaces along $\gamma$ and uses these linear subspaces to approximate the phase resetting curve.

Some preliminaries: the limiting map $\bar{F}_{T}=\lim _{n \rightarrow \infty} F_{T+n T_{0}}$ can be characterized abstractly by the equation

$$
\bar{F}_{T}=\pi_{\mathrm{ss}} \circ \phi_{T} \circ K_{A}=\phi_{T} \circ \pi_{\mathrm{ss}} \circ K_{A},
$$

where $\pi_{\mathrm{ss}}(x)=y$ if and only if $x \in W_{\mathrm{ss}}(y)$, i.e. $\pi_{\mathrm{ss}}: B(\gamma) \rightarrow \gamma$ maps the basin of $\gamma$ onto the limit cycle $\gamma$ along strong-stable manifolds. The abstract notations (and notions) have their uses: the projection $\pi_{\mathrm{ss}}$ encapsulates the properties of the strong-stable manifolds, e.g. by definition, the strong stable manifold $W_{\mathrm{ss}}\left(\pi_{\mathrm{ss}}(x)\right)$ passes through $x$ for any $x \in B(\gamma)$, and the nullspace of the Jacobian matrix $D \pi_{\mathrm{ss}}(x)$ is precisely the tangent space of the strong stable manifold $W_{\mathrm{ss}}\left(\pi_{\mathrm{ss}}(x)\right)$ at $x .^{6}$

\section{Algorithm (Phase resetting curves via stable subspaces).}

1. Estimate the period $T_{0}$ of the limit cycle $\gamma$ by numerically solving the unforced equations starting with a point on or near $\gamma$.

2. Discretize $\gamma$ by subdividing the time interval $\left[0, T_{0}\right)$ into $N$ intervals and computing the corresponding points $x_{i} \in \gamma$. Fix an arbitrary reference point $x_{0}$ on $\gamma$ so that each point on $\gamma$ can be assigned a unique phase $\theta \in\left[0, T_{0}\right)$.

3. For each point $x_{i}$ computed in the previous step, compute the Jacobian $D H\left(x_{i}\right)$ of the HodgkinHuxley flow field $H$ at that point.

4. Using the results of the previous two steps, solve

$$
\begin{aligned}
\dot{x} & =-H(x), \\
\dot{\xi} & =\eta-\langle\eta, \xi\rangle \xi, \\
\eta & =D H(x)^{T} \xi,
\end{aligned}
$$

using the grid points $\left\{x_{i}\right\}$ computed in the previous steps. The $\dot{x}$ part of the equation above is clearly numerically unstable, but that is not a problem because we have already have a numerical representation of $\gamma$.

The equations above are a variant of the usual method for computing Lyapunov exponents [13]. They preserve the length of $\xi(t)$, though in practice, it is necessary to rescale $\xi(t)$ to ensure that this constraint is maintained. As $t \rightarrow \infty, \xi(t)$ becomes orthogonal to the strong-stable linear subspace $E_{\mathrm{ss}}(x(t))$ of $\gamma$. The subspace $E_{\mathrm{ss}}(x(t))$ is tangent to the strong-stable manifold $W_{\mathrm{ss}}(x(t))$ at $x(t) .^{7}$

\footnotetext{
${ }^{6}$ Note that the commutation relation $\phi_{t} \circ \pi_{\mathrm{ss}}=\pi_{\mathrm{ss}} \circ \phi_{t}$ expresses the invariance of the strong-stable foliation under $\phi_{t}$. The map $K_{A}$, in general, has nothing to do with the flow and does not commute with the other maps.

${ }^{7}$ These equations can be generalized to the following:

$$
\begin{aligned}
& \dot{x}=-H(x) \\
& \dot{\xi}_{i}=\eta_{i}-\left\langle\eta_{i}, \xi_{i}\right\rangle \xi_{i}-\sum_{j<i}\left(\left\langle\xi_{i}, \dot{\xi}_{j}\right\rangle+\left\langle\eta_{i}, \xi_{j}\right\rangle\right) \xi_{j}
\end{aligned}
$$$$
\eta_{i}=D H(x)^{T} \xi_{i}
$$

If the vectors $\left(\xi_{i}\right)$ form an orthonormal basis at $t=0$, then the equations will guarantee that $\left(\xi_{i}(t)\right)$ are orthonormal for all $t>0$. Again, it will be necessary to perform Gram-Schmidt orthogonalizations periodically to maintain this constraint. The vector $\xi_{1}(t)$, as before, converges to a vector orthogonal to $E_{\mathrm{ss}}(x(t))$. So $\left(\xi_{2}(t), \xi_{3}(t), \xi_{4}(t)\right)$ span $E_{\mathrm{ss}}(x(t))$. Similarly, the vectors $\left(\xi_{3}(t), \xi_{4}(t)\right)$ span the subspace consisting of the 2 fastest contracting directions, and $\left(\xi_{4}(t)\right)$ spans the fastest contracting direction.
} 
5. Using Eq. 18 in combination with the linear subspaces computed in the previous step, we can now approximate the phase resetting curve. Start with a point $x \in \gamma$ and compute $\Phi_{t}\left(K_{A}(x)\right)$ for increasing $t$. Let $t_{0}>0$ be the minimum positive time at which $\Phi_{t_{0}}\left(K_{A}(x)\right)$ has (i) returned to a small, fixed neighborhood of $\gamma$ (in this paper this is chosen to be a neighborhood of distance $10^{-4}$ around $\gamma$ ); and (ii) $\Phi_{t_{0}}\left(K_{A}(x)\right)$ lies within one of the pre-computed linear subspaces $E_{\mathrm{ss}}\left(x_{*}\right)$ for some point $x_{*}$. Let $\theta_{*}$ denote the phase of the point $x_{*}$. Then the new phase of the system is $\left(T+\theta_{*}-t_{0}\right)\left(\bmod T_{0}\right)$.

6. Proceed to the next grid point and repeat.

7. When the derivative of the phase resetting curve becomes large or infinite, it may be necessary to adaptively generate the grid points on which the curve is evaluated. Generally speaking, the grid $\left\{x_{i}\right\}$ constructed in Step 2 need not equal the grid $\left\{x_{i}^{\prime}\right\}$ on which the phase resetting curve is evaluated. In particular, the grid $\left\{x_{i}^{\prime}\right\}$ can be adaptively chosen to ensure that $\left|\hat{f}_{a}\left(x_{i+1}^{\prime}\right)-\hat{f}_{a}\left(x_{i}^{\prime}\right)\right| \leq \epsilon$, where $\hat{f}_{a}$ denotes the computed phase resetting curve and $\epsilon$ is a fixed number, in this paper usually 0.1 . This adaptive mechanism provides a way to detect discontinuities in $f_{T}$.

\section{Acknowledgements}

It is also a pleasure to thank Lai-Sang Young, Eric Shea-Brown, Adi Rangan, Charlie Peskin, John Rinzel, and Louis Tao for many helpful and stimulating conversations. I am grateful to the referees for their help in improving the exposition and for pointing out relevant references. This work is partially supported by an NSF Postdoctoral Fellowship.

\section{References}

[1] K. Aihara And G. Matsumoto, Chaotic oscillations and bifurcations in squid giant axons, in Chaos, Nonlinear Science: Theory and Applications, Manchester University Press, 1986.

[2] J. C. Alexander, E. J. Doedel, And H. G. Othmer, On the resonance structure in a forced excitable system, SIAM Journal on Applied Mathematics, 50 (1990), pp. 1373-1418.

[3] E. N. BEST, Null space in the Hodgkin-Huxley equations: a critical test, Biophysical Journal, 27 (1979), pp. 87-104.

[4] E. BROWN, J. MOEHLIS, AND P. HOLMES, On the phase reduction and response dynamics of neural oscillator populations, Neural Computation, 16 (2004), pp. 673-715.

[5] J. CRonin, Mathematical Aspects of Hodgkin-Huxley Neural Theory, Cambridge University Press, 1987.

[6] S. DoI, A chaotic map with a flat segment can produce a noise-induced order, Journal of Statistical Physics, 55 (1989), pp. 941-964.

[7] G. B. Ermentrout, Simulating, Analyzing, and Animating Dynamical Systems: A Guide to XPPAUT for Researchers and Students, vol. 14 of Software, Environments, and Tools, SIAM, 2002.

[8] G. B. ERmentrout AND N. KopelL, Multiple pulse interactions and averaging in systems of coupled neural oscillators, Journal of Mathematical Biology, 29 (1991), pp. 195-217. 
[9] G. B. ERmentrout, M. PASCAL, AND B. GUTKIN, The effects of spike frequency adaptation and negative feedback on the synchronization of neural oscillators, Neural Computation, 13 (2001), pp. 1285-1310.

[10] K. Geist, U. Parlitz, AND W. Lauterborn, Comparison of different methods for computing Lyapunov exponents, Progress of Theoretical Physics, 83 (1990), pp. 875-893.

[11] L. Glass AND M. C. MaCKey, From Clocks to Chaos: The Rhythms of Life, Princeton University Press, 1988.

[12] J. GUCKENHEIMER, Isochrons and phaseless sets, Journal of Theoretical Biology, 1 (1974), pp. 259-273.

[13] J. GuCKenheimer AND P. Holmes, Nonlinear Oscillations, Dynamical Systems, and Bifurcations of Vector Fields, Springer-Verlag, 1983.

[14] R. GUtTMAn, L. FeLdMAN, AND E. JAKOBSSON, Frequency entrainment of squid axon membrane, Journal of Membrane Biology, 56 (1980), pp. 9-18.

[15] H. HAYASHI, S. ISHIZUKA, AND K. HIRAKAWA, Chaotic response of the pacemaker neuron, Journal of the Physical Society of Japan, 54 (1985), pp. 2337-2346.

[16] H. Hayashi, S. Ishizuka, M. OHTA, AND K. HirakaWA, Chaotic behavior in the onchidium giant neuron under sinusoidal stimulation, Physics Letters, 88A (1982), pp. 435-438.

[17] H. HAYASHI, M. NAKAO, AND K. HIRAKAWA, Entrained, harmonic, quasiperiodic and chaotic responses of the self-sustained oscillation of nitella to sinusoidal stimulation, Journal of the Physical Society of Japan, 52 (1983), pp. 344-351.

[18] A. L. HodgKIn AND A. F. HuxLey, A quantitative description of membrane current and its application to conduction and excitation in nerve, Journal of Physiology, 117 (1952), pp. 500-544.

[19] A. V. Holden, The response of excitable membrane models to a cyclic input, Biology and Cybernetics, (1975), pp. 1-7.

[20] D. H. Hubel, Eye, Brain, and Vision, Scientific American Library, 1988.

[21] J. D. Hunter, J. G. Milton, P. J. Thomas, And J. D. Cowan, Resonance effect for neural spike time reliability, Journal of Neurophysiology, 80 (1998).

[22] F. LEDRAPPIER AND L.-S. YOUNG, Entropy formula for random transformations, Probability Theory and Related Fields, 80 (1988), pp. 217-240.

[23] Z. F. MAINEN AND T. J. SEJNOWSKI, Reliability of spike timing in neocortical neurons, Science, 268 (1995), pp. 1503-1506.

[24] G. Matsumoto, K. Aihara, Y. Hanyu, N. Takahashi, S. Yoshizawa, and J. ichi Nagumo, Chaos and phase locking in normal squid axons, Physics Letters A, 123 (1987), pp. 162-166.

[25] G. Matsumoto, N. TAKAhashi, AND Y. HANYU, Chaos, phase locking and bifurcation in normal squid axons, in Chaos in Biological Systems, Plenum, 1987.

[26] H. NAKaO, K. SUKe Arai, K. Nagai, Y. Tsubo, And Y. KURAmoto, Synchrony of limit-cycle oscillators induced by random external impulses, Physical Review E, 72 (2005). 
[27] A. OKSASOGLU AND Q. WANG, Strange attractors in periodically-kicked Chua's circuit, International Journal of Bifurcation and Chaos, (to appear).

[28] S. A. OpRISAN AND C. C. CANAVIER, The influence of limit cycle topology on the phase resetting curve, Neural Computation, 14 (2002), pp. 1027-1057.

[29] K. Pakdaman And S. TANabe, Random dynamics of the Hodgkin-Huxley neuron model, Physical Review E, 64 (2001).

[30] W. H. Press, B. P. Flannery, S. A. Teukolsky, and W. T. Vetterling, Numerical Recipes in C, Cambridge University Press, 1992.

[31] F. RIEKE, Spikes: Exploring the Neural Code, MIT Press, c1997.

[32] T. TAKabe, K. Aihara, And G. Matsumoto, Response characteristics of the Hodgkin-Huxley equations to pulse-train stimulation, Transactions of IEICE Japan (Japanese edition), J71-A (1988), pp. 744-750.

[33] Q. WANG AND L.-S. YOUNG, Strange attractors with one direction of instability, Communications in Mathematical Physics, 218 (2001).

[34] _ From invariant curves to strange attractors, Communications in Mathematical Physics, 225 (2002).

[35] _ Strange attractors in periodically-kicked limit cycles and Hopf bifurcations, Communications in Mathematical Physics, 240 (2003).

[36] — - Toward a theory of rank-1 attractors, preprint, (2004).

[37] T. L. Williams AND G. BOWTELL, The calculation of frequency-shift functions for chains of coupled oscillators, with application to a network model of the lamprey locomotor pattern generator, Journal of Computational Neuroscience, 4 (1997), pp. 47-55.

[38] A. WinfreE, The Geometry of Biological Time, Second Edition, Springer-Verlag, 2000.

[39] L.-S. YOUNG, Ergodic theory of differentiable dynamical systems, in Real and Complex Dynamics, NATO ASI Series, Kluwer Academic Publishers, 1995, pp. 293-336.

[40] G. ZASLAVSKY, The simplest case of a strange attractor, Physics Letters, 69A (1978), pp. 145-147.

[41] C. ZHOU AND J. KURTHS, Noise-induced synchronization and coherence resonance of a Hodgkin-Huxley model of thermally sensitive neurons, Chaos, 13 (2003), pp. 401-409. 


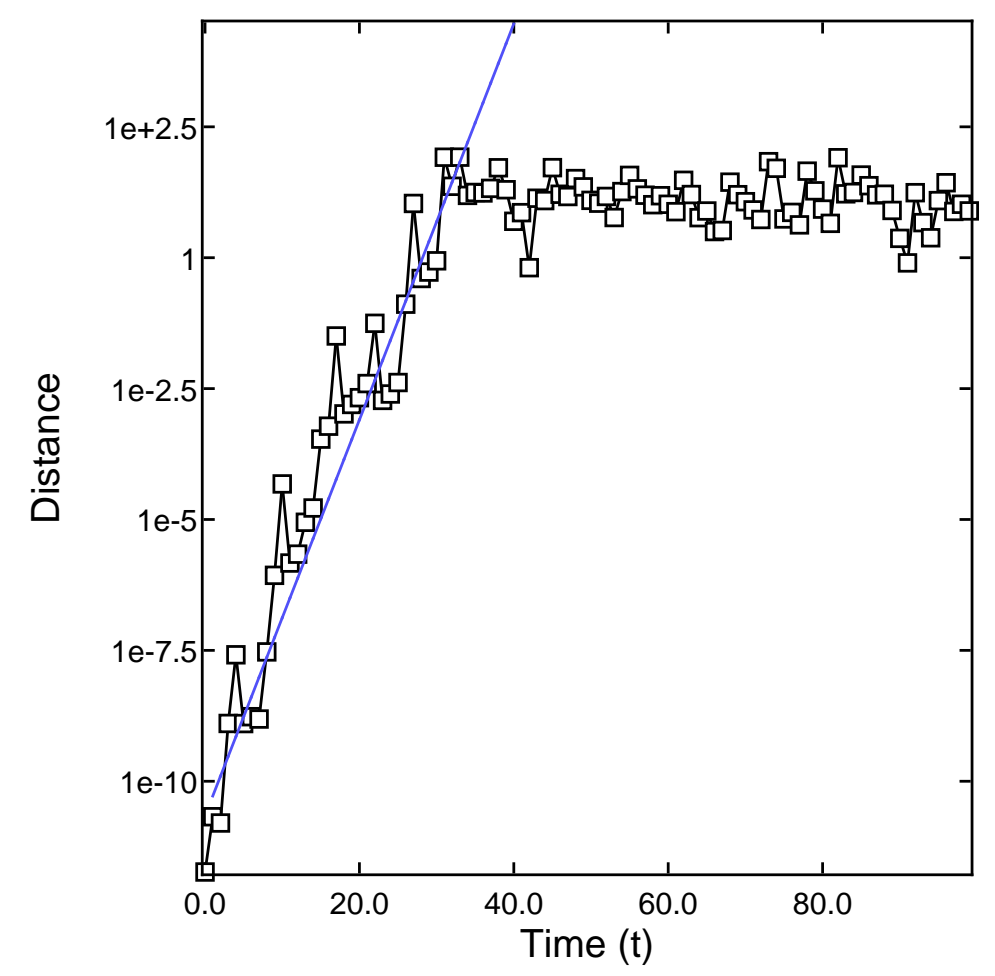

